\title{
STAT4 is expressed in neutrophils and promotes antimicrobial immunity
}

\author{
Pegah Mehrpouya-Bahrami, ${ }^{1,2}$ Alina K. Moriarty, ${ }^{3}$ Paulo De Melo, ${ }^{4}$ W. Coles Keeter, ${ }^{3}$ \\ Nada S. Alakhras, ${ }^{5}$ Andrew S. Nelson, ${ }^{1,2}$ Madeline Hoover, ${ }^{2}$ Maria S. Barrios, ${ }^{2}$ Jerry L. Nadler, ${ }^{6}$ \\ C. Henrique Serezani, ${ }^{4,7}$ Mark H. Kaplan,, ${ }^{1,2,5}$ and Elena V. Galkina ${ }^{3}$ \\ 1Department of Microbiology and Immunology and ${ }^{2}$ Herman B Wells Center for Pediatric Research, Department of \\ Pediatrics, School of Medicine, Indiana University, Indianapolis, Indiana, USA. ${ }^{3}$ Department of Microbiology and Molecular \\ Cell Biology, Eastern Virginia Medical School, Norfolk, Virginia, USA. ${ }^{4}$ Division of Infectious Diseases, Department of \\ Medicine, Vanderbilt University Medical Center, Nashville, Tennessee, USA. ${ }^{5}$ Department of Biochemistry and Molecular \\ Biology, School of Medicine, Indiana University, Indianapolis, Indiana, USA. 'Departments of Medicine and Pharmacology, \\ School of Medicine, New York Medical College, Valhalla, New York, USA. ${ }^{7}$ Department of Pathology, Microbiology and \\ Immunology, Vanderbilt University Medical Center, Nashville, Tennessee, USA.
}

Signal transducer and activator of transcription 4 (STAT4) is expressed in hematopoietic cells and plays a key role in the differentiation of T helper 1 cells. Although STAT4 is required for immunity to intracellular pathogens, the T cell-independent protective mechanisms of STAT4 are not clearly defined. In this report, we demonstrate that STAT4-deficient mice were acutely sensitive to methicillin-resistant Staphylococcus aureus (MRSA) infection. We show that STAT4 was expressed in neutrophils and activated by IL-12 via a JAK2-dependent pathway. We demonstrate that STAT4 was required for multiple neutrophil functions, including IL-12-induced ROS production, chemotaxis, and production of the neutrophil extracellular traps. Importantly, myeloid-specific and neutrophil-specific deletion of STAT4 resulted in enhanced susceptibility to MRSA, demonstrating the key role of STAT4 in the in vivo function of these cells. Thus, these studies identify STAT4 as an essential regulator of neutrophil functions and a component of innate immune responses in vivo.

Authorship note: CHS, MHK, and EVG are co-senior authors. PMB, AKM, and PDM contributed equally to this work.

Conflict of interest: The authors have declared that no conflict of interest exists.

Copyright: (c) 2021, MehrpouyaBahrami et al. This is an open access article published under the terms of the Creative Commons Attribution 4.0 International License.

Submitted: June 15, 2020

Accepted: June 3, 2021

Published: July 22, 2021

Reference information: JCl Insight 2021;6(14):e141326.

https://doi.org/10.1172/jci.

insight.141326.

\section{Introduction}

Signal transducer and activator of transcription (STAT) proteins are a family of factors implicated in various biological processes, including the induction of genes involved in cell differentiation (1). STAT4, activated downstream of IL-12, is the only STAT that shows tissue-restricted expression, with mRNA constitutively expressed in lymphoid cells and inducibly expressed in monocytes and macrophages (2-4). STAT4 is required for all known IL-12 biological functions, including the induction of IFN- $\gamma$ and the promotion of Thelper type 1 (Th1) differentiation $(5,6)$. Single nucleotide polymorphisms (SNPs) of the STAT4 gene have been associated with asthma, Sjögren's syndrome, rheumatoid arthritis, and systemic lupus erythematosus (7). In models of infection and autoimmunity, STAT4 is a critical component in developing inflammation (8). STAT4-deficient mice are susceptible to infection with intracellular pathogens, have decreased delayed-type hypersensitivity (DTH) responses (8), and have attenuated T cell responses $(5,6)$. In contrast, STAT4-deficient mice are refractory to the induction of inflammatory conditions, including colitis, arthritis, diabetes, myocarditis, and experimental autoimmune encephalitis (7). Thus, STAT4 is required for inflammatory immunity.

Many of the studies on STAT4 function have focused on T cells. Only a few reports have investigated STAT4's role in macrophages and dendritic cells. The homeostatic numbers of myeloid cells are normal in the absence of STAT4, despite alterations in hematopoietic progenitor cell numbers (9). In human monocytes and mouse dendritic cells, STAT4 expression is induced with maturation, and rheumatoid synovia-isolated macrophages express STAT4 $(3,4)$. Macrophage stimulation with either IL-12 or type I IFNs results in STAT4-dependent expression of Ifng, Tnfa, and Nos2 $(3,4,10,11)$. Thus, STAT4 is clearly functional in monocytes and macrophages. While STAT4 is involved in shaping inflammatory responses, it has not been defined whether STAT4 is expressed in neutrophils or whether its activation would affect neutrophil functions in normal and pathological conditions.

Notably, there is growing evidence that STAT4 is functionally important in innate cells. SNPs in the STAT4 gene are associated with diseases with a clear link to innate immunity $(7,8)$. Moreover, patients with mutations 
in IL-12 signaling become susceptible to bacterial infections $(12,13)$. Infection of Stat ${ }^{-1}$ mice with Salmonella typhimurium, Klebsiella pneumonia, and Mycobacterium tuberculosis results in a bacterial burden that is orders of magnitude greater than controls (8). Godshall and colleagues demonstrated that STAT4 is required for antibacterial defense during polymicrobial peritonitis in the cecal ligation and puncture model (14). Interestingly, STAT4 deficiency is also linked to impaired production of proinflammatory cytokines in inflammation induced by Pseudomonas aeruginosa ( $P$. aeruginosa) in BALB/c mice but was not implicated in bacterial clearance (15). STAT4 expression in innate lymphoid cells contributes to Listeria monocytogenes immunity (16). Overall, while there were some indications suggesting the involvement of the IL-12/STAT4 pathway in the host defense, the cells responsible for STAT4-dependent antibacterial immunity and the cellular processes that control antibacterial responses have not been identified. In this report, using STAT4-conditional mutant mice and bacterial infection in vivo, we define STAT4-dependent neutrophil functions during infection.

\section{Results}

STAT4 is expressed in neutrophils, is activated by IL-12, and regulates gene expression. Much of the research on IL-12and IFN- $\alpha$-dependent functions of STAT4 has been performed in T and NK cells (8); however, evidence suggests that STAT4 is also expressed in activated monocytes, macrophages, and dendritic cells. Additionally, an essential role of the STAT4 pathway has been demonstrated in both the cecal ligation and puncture model (14) and $P$. aeruginosa-induced inflammation (15). The rapidity of the response observed during infections suggested that STAT4 function in innate immune cells was required for this response, but specific subpopulations of STAT4-dependent innate immune cells that are responsible for the phenotype have not been identified.

Neutrophils are a critical early component of innate immunity and play a central role in defense against various bacterial pathogens, such as $S$. aureus (17). To date, no reports demonstrate STAT4 expression or function in neutrophils. Thus, we explored whether neutrophils express STAT4 and respond to IL-12, a key STAT4-activating cytokine (8). Murine neutrophils isolated from the bone marrow (BM) of WT mice clearly expressed STAT4 (Figure 1A). Notably, IL-12 treatment induced time-dependent phosphorylation of STAT4 in BM neutrophils (Figure 1A). To further understand how IL-12 induces STAT4 phosphorylation in neutrophils, we next focused on intracellular signal transduction processes initiated by type I cytokines that utilize various JAK proteins to activate STAT4 (18). We demonstrated that JAK2, an upstream signaling kinase of STAT4, underwent rapid phosphorylation upon IL-12 stimulation (Figure 1B). To confirm that IL-12 induces JAK2-dependent activation of STAT4, we used gandotinib (LY2784544), a potent, selective small-molecule JAK2 inhibitor that has dose-dependent selectivity for the JAK2 (19). As shown in Figure 1C, the treatment of IL-12-stimulated neutrophils with gandotinib diminished STAT4 phosphorylation, suggesting that IL-12 signals through the canonical JAK2/STAT4 pathway in neutrophils.

We next utilized PCR arrays that profile 84 genes related to antimicrobial response to investigate the transcriptional outcomes of STAT4 functionality in IL-12-induced neutrophil activation. The major effect of IL-12 treatment for WT neutrophils was in the upregulation of signal transduction pathways associated with inflammation, apoptosis, and cytokine signaling (Il6, Ripk1, Tirap, Ticam1), with the main effect on Jun expression. Unexpectedly, STAT4 deficiency in neutrophils resulted in upregulation of some genes responsible for the induction of inflammation, such as Lbp and Tlr2, 4, and 5 (TLR activation); Card 9 and Tnfrsfla (positive regulation of apoptosis, NF-кB, and ROS production); Lyz2 and Prtn3a (granule formation/release); Pstpip1 and Mefv (cytoskeleton and autophagy, respectively); Jun and Mapk2k3, Mapk14, and Mapk3 (signal transduction); and Nlrpla and Illb (inflammasome formation) at the basal state (Figure 1D). In contrast, genes related to TLRdependent regulation of innate immunity (Nod1, Infbl) were diminished in Stat $4^{-1}$ versus WT neutrophils (Figure 1D). Further comparison of IL-12-treated Stat4 ${ }^{-/-}$and vehicle-treated control Stat4 ${ }^{-/}$neutrophils showed that IL-12 also had additional STAT4-independent effects via regulation of inflammatory pathways (Ifnb1; Irak3; Irf5; Nod1; Mapk1, 3, and 14; and Mefv). Interestingly, a comparison of IL-12-activated Stat4 ${ }^{-1-}$ and WT neutrophils demonstrated differences in the induction of key proinflammatory genes (Casp8, Map3k7, Mapk1, $N f k b 1$, and Il18) responsible for NF- $\mathrm{BB}$ and inflammasome pathway activation (20). Unexpectedly, IL-12-treated Stat $4^{-/}$neutrophils also demonstrated upregulation of Cxcl1, Card9, Lyz2, Nlrp1a, Prtn3, Sipi, and Tnfrsfla expression compared with IL-12-treated WT neutrophils (Figure 1D), suggesting a hyperactivated phenotype of Stat4 ${ }^{-1}$ neutrophils. These data support the conclusion that IL-12/STAT4 signaling is active in neutrophils and reveal a complex role of the IL-12/STAT4 axis in the regulation of proinflammatory gene expression.

STAT4 supports chemotaxis, migration, and IL-12-dependent ROS production and bacterial killing in neutrophils. To avoid the complications of examining myeloid cell function in Stat4 germline mutant mice, we generated 
A
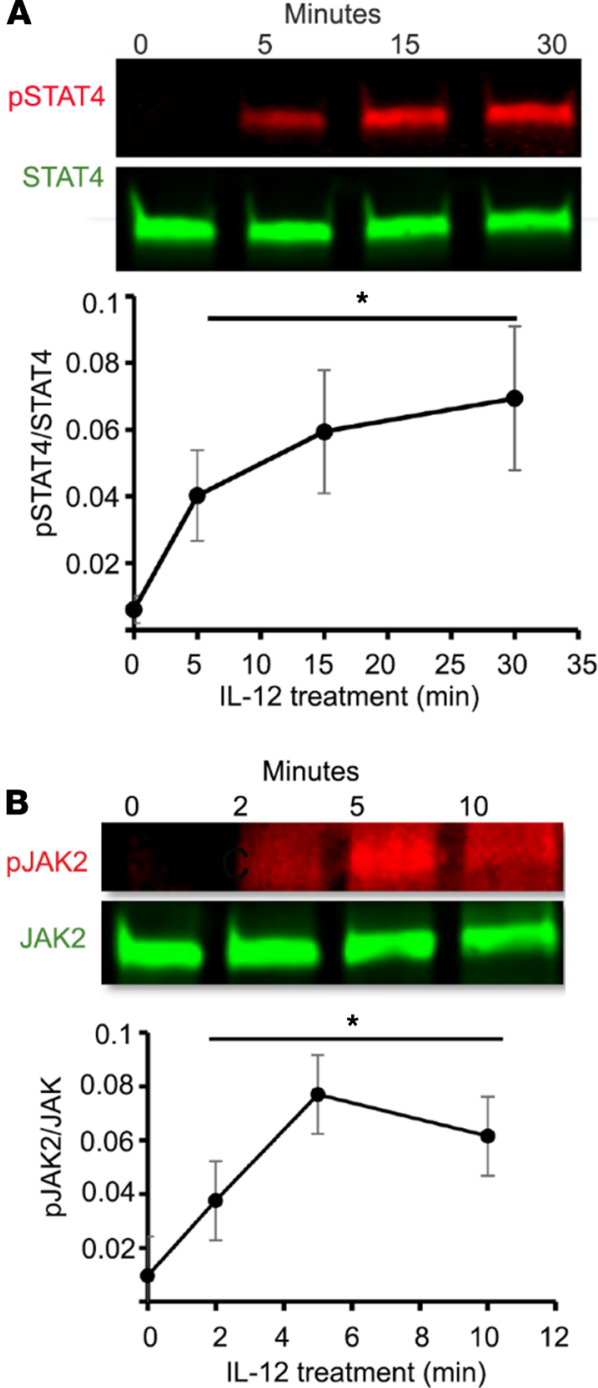

D

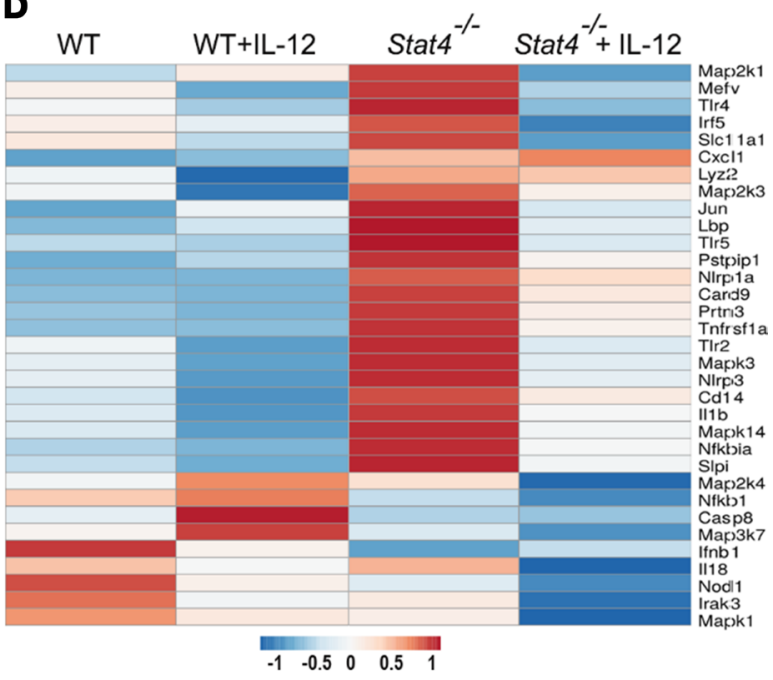

C
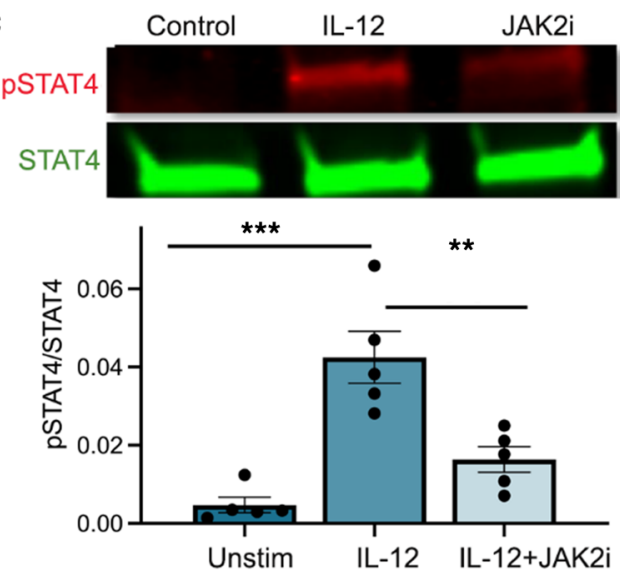

Figure 1. STAT4 is expressed in neutrophils, is activated by IL-12, and regulates gene expression profile of neutrophils. BM neutrophils were treated with IL-12 (40 ng/mL) for indicated time points. (A) The expression of phosphorylated STAT4/STAT4 (p-STAT4/STAT4) or (B) p-JAK/JAK was examined by Western blot. Representative image from 3 independent experiments ( $n=3-5$ mice total). (C) BM neutrophils were either pretreated with the JAK2 inhibitor (JAK2i) gandotinib $(1 \mu \mathrm{M})$ or left untreated, and 15 minutes later, all samples were stimulated with IL-12 for 30 minutes, and expression of $p$-STAT4/STAT4 was determined by Western blotting at the indicated time points ( $n=5$ mice/per group in 3 independent experiments). (D) Heatmaps of real-time RT ${ }^{2}$ Profiler PCR Array showing differential expression of genes between WT and Stat4 ${ }^{-/}$IL-12-treated and -untreated samples. Differences between groups were assessed by 2-tailed Student's $t$ test $\left({ }^{*} P<0.05\right)$, using the RT ${ }^{2}$ Profiler PCR Array Analysis ( $n=3$ samples/group). Data are mean \pm SEM; ${ }^{*} P<0.05$, ${ }^{* *} P<0.01,{ }^{* *} P<0.001$ using 1-way ANOVA followed by Tukey-Kramer post hoc test.

a Stat $4^{\text {I/f }}$ mouse model and crossed to either myeloid cell-specific (Lys $\left.M^{* r}\right)$ or neutrophil-specific $\left(S 100 A 8^{* r}\right)$ Cre-transgenic mice (Supplemental Figure 1, A and B; supplemental material available online with this article; https://doi.org/10.1172/jci.insight.141326DS1). Deletion of STAT4 was observed in neutrophils and

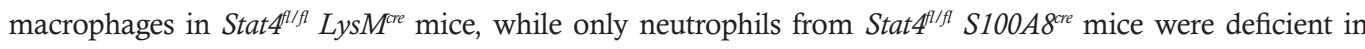
STAT4 expression (Supplemental Figure 1C). Importantly, Stat $4^{\text {I/f }} L y s M^{\text {rer }}$ mice had normal development of myeloid cells in secondary lymphoid tissues and blood, and Th1 differentiation was normal in T cell cultures from Stat $4^{\nexists / f} L y s M^{\text {rer }}$ mice (Supplemental Figure 1, D-F). In line with specific STAT4 deletion in myeloid cells, we observed diminished activation of STAT4 in methicillin-resistant Staphylococcus aureus- (MRSA-activated) and IL-12-activated BM-derived macrophages (BMDMs) and reduced induction of known STAT4 target genes in cultured BMDMs (Supplemental Figure 1, G and H).

Because neutrophils are the first responders to fight infections, these cells must migrate efficiently to the site of infection to eliminate invading pathogens (17). To test how STAT4 deficiency affects neutrophil recruitment to sites of inflammation, we first examined chemotaxis of neutrophils to CXCL1 in Transwell assays. 
A

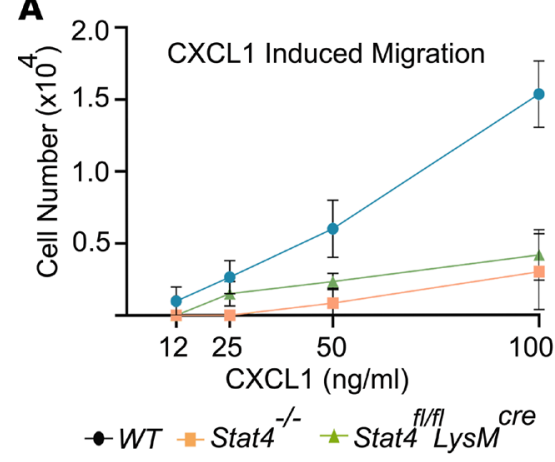

D

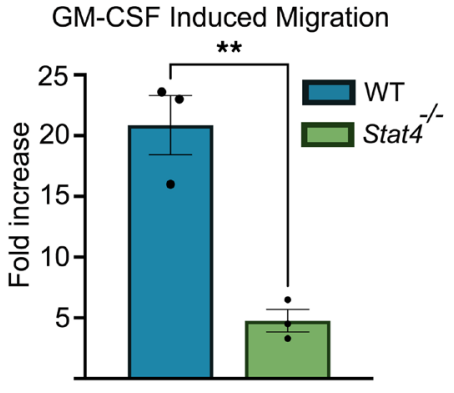

B

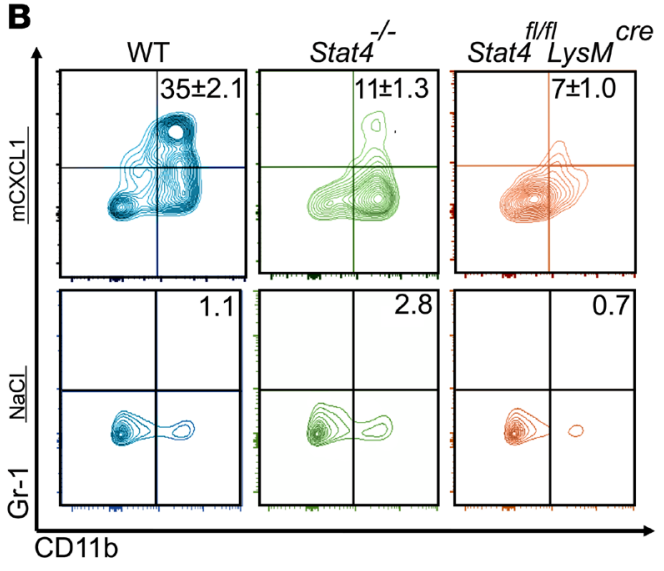

E

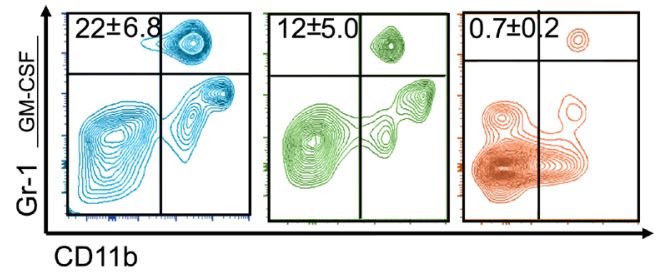

C
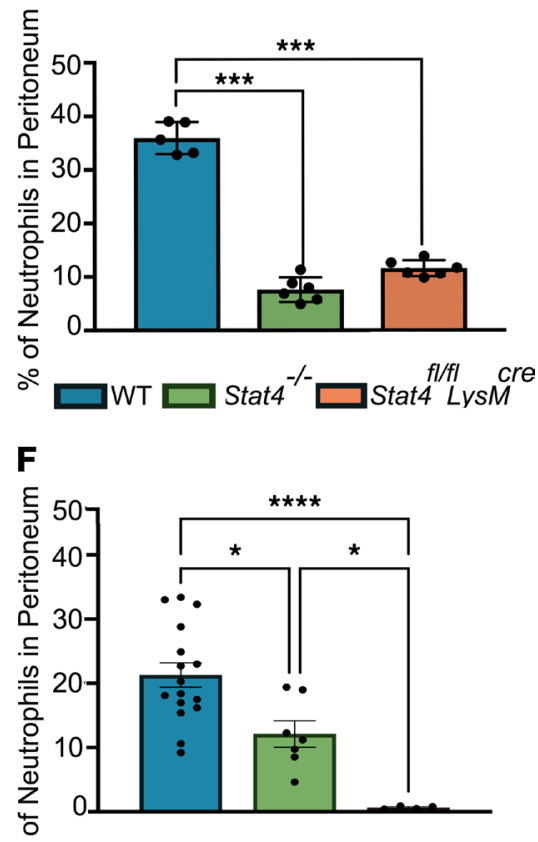

Figure 2. STAT4 supports chemotaxis and migration of neutrophils. (A) CXCL1-induced chemotaxis of WT, Stat4 $4^{-/-}$, and Stat $4^{f / f l}$ LysM $M^{\text {cre }}$ neutrophils in a Transwell assay ( $n=3 /$ group in 2 independent experiments). (B and C) WT, Stat4 ${ }^{-/}$, and Stat4 ${ }^{f / f}$ Lys $M^{\text {cre }}$ mice were injected i.p. with CXCL1 or 0.9\% NaCl. After 4 hours, percentage of extravasated neutrophils in the lavage were determined $\left(n=5-6 /\right.$ group in 4 independent experiments). ${ }^{* * *} P<0.001$ by 1 -way ANOVA followed by post hoc test. (D) GM-CSF-induced chemotaxis of WT and Stat4 ${ }^{-/-}$neutrophils in a Transwell assay ( $n=3 / \mathrm{group}$ in 3 independent experiments). ${ }^{*} P<0.05$ using paired 2-tailed Student's $t$ test. (E and F) WT, Stat4 ${ }^{-/}$, and Stat $4^{f / f l}$ Lys $M^{c r e}$ mice were injected i.p. with recombinant murine $\mathrm{CM}-\mathrm{CSF}$ (rmGM-CSF), and 4 hours later percentage of extravasated neutrophils in the lavage was determined ( $n=5-17 / g r o u p$ in 7 independent experiments). ${ }^{* *} P<0.001$ using Welch's 1-way ANOVA test with Dunnett's post hoc test.

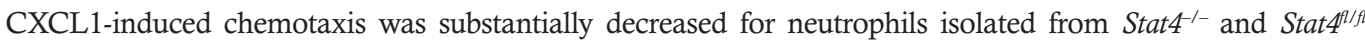
Lys $M^{\text {cre }}$ mice (Figure 2, A-C). We next investigated how STAT4 deletion affects neutrophil recruitment in vivo. We injected CXCL1 or saline into the peritoneal cavity (PC) of WT, Stat4 ${ }^{-/}$, and Stat $4^{\text {I/fl }}$ LysM $^{\text {rre }}$ mice. After 4

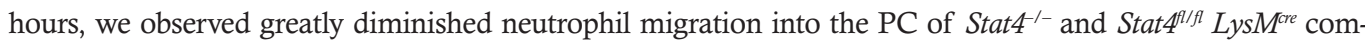
pared with WT mice (Figure 2, B and C), indicating that STAT4 promotes neutrophil recruitment. GM-CSF regulates neutrophil recruitment in vivo (21). To further test the role of STAT4 in the regulation of chemotaxis, we performed GM-CSF-induced transmigration assays. We detected an approximately 4-fold decrease in migration between Stat4 ${ }^{--}$and WT neutrophils (Figure 2D). Importantly, these data were further confirmed by results from GM-CSF-induced recruitment of neutrophils into the $\mathrm{PC}$ in vivo (Figure 2, E and F).

We then asked whether additional functions of neutrophils were dependent on the IL-12/STAT4 axis. As shown in Figure 3A, IL-12 induced substantial ROS production in neutrophils. In contrast, ROS production was almost abolished in Stat $4^{A / f l}$ LysM $M^{\text {cre }}$ or Stat $4^{f / f l}$ S100A8 $8^{\text {re }}$ neutrophils (Figure 3B). IL-12-induced ROS production was not due to increased apoptosis (Supplemental Figure 2, A and B). While IL-12 is an essential component that initiates STAT4 activation, we also sought to test whether other stimuli might induce STAT4-dependent activation of neutrophils. We, therefore, tested a role of STAT4 in TLR-dependent responses. Interestingly, LPS-induced ROS production was also at least partially STAT4 dependent (Figure 3, C and D). Neutrophils are capable of secreting a broad spectrum of cytokines and chemokines. Evidence demonstrates that neutrophils contain cytokines, particularly IL-12 and IL-6, in preformed granules and can quickly release these intracellular stores upon stimulation (22). Therefore, we sought to explore whether TLR-dependent neutrophil activation induces IL-12 release by neutrophils, activation of STAT4, and a subsequent increase in ROS production. The presence of neutralizing Abs to IL-12 abolished effects of LPS stimulation and ROS production and mimicked the response seen with STAT4-deficient neutrophils (Figure 3, C and D). These results suggest that TLR-induced ROS production occurs at least partially via an IL-12/STAT4-dependent pathway.

The neutrophil is equipped with several pathways for bacterial killing, including the generation of ROS and release of granules containing proteases and antimicrobial peptides. Because STAT4 regulated ROS 
A

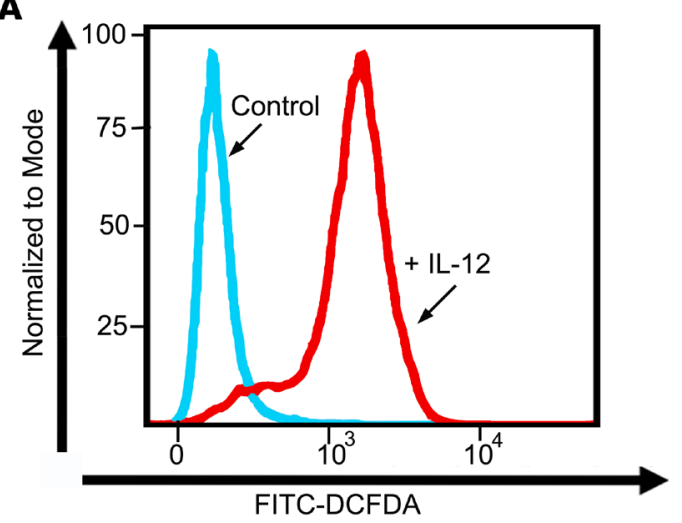

C

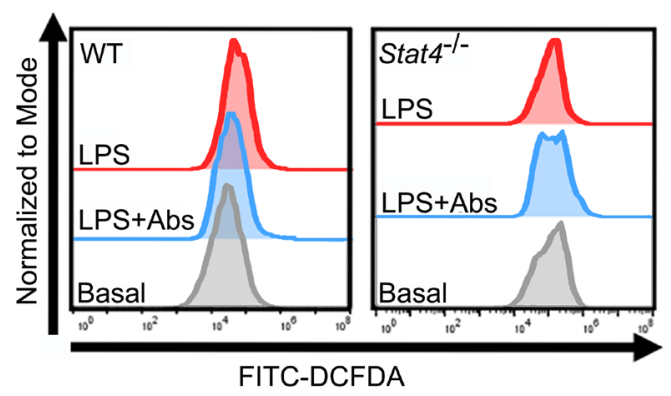

B

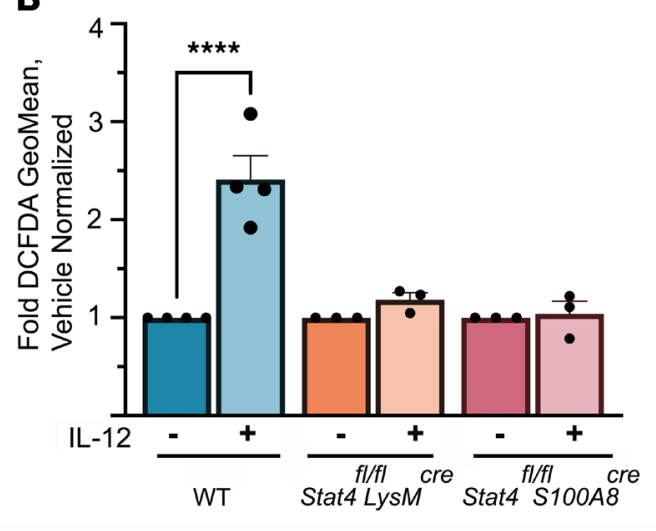

D

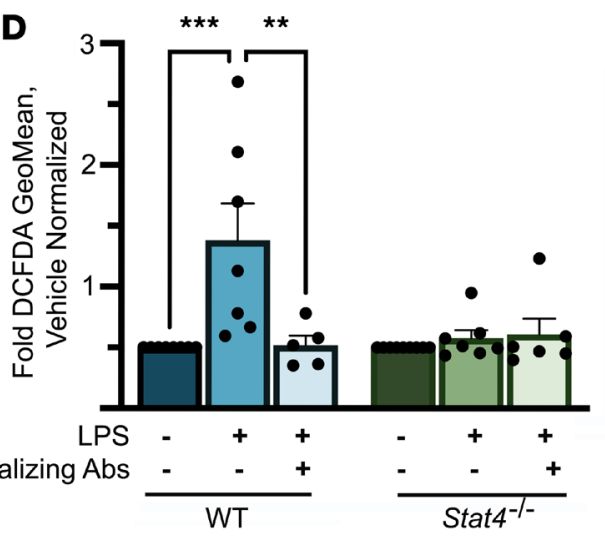

$\mathbf{E}$

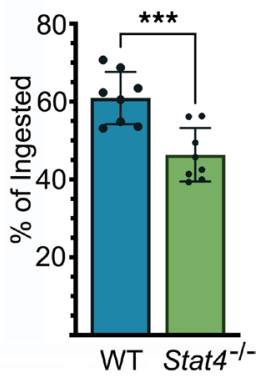

IL-12 neutralizing Abs

Figure 3. Neutrophil ROS production and bacterial killing are STAT4 dependent. (A and B) WT, Stat4 ${ }^{-/-}$, and Stat4//fl LysM ${ }^{c r e}$ BM leukocytes were labeled with FITC-DCFDA, stimulated with IL-12 (40 ng/mL), stained with anti-CD11b, anti-Ly6C Abs, and analyzed for ROS production by FACS 14 hours later ( $n=3-4 /$ group in 2 independent experiments). FITC-DCFDA was used to measure ROS levels. (C and D) FITC-DCFDA-labeled WT, Stat4 ${ }^{-/-}$, and Stat4//fl LysM ${ }^{\text {cre }}$ BM leukocytes were

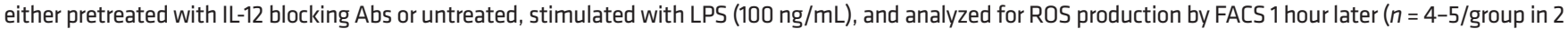
independent experiments). (A-D) ${ }^{* * *} P<0.001$ using 1-way ANOVA followed by Tukey-Kramer post hoc test. (E) Bacterial killing of GFP-tagged MRSA by neutrophils from WT and Stat4 ${ }^{-/-}$mice as described in Phagocytosis and killing assays. ${ }^{* *} P<0.01,{ }^{* *} P<0.001,{ }^{* * *} P<0.0001$ using paired 2 -tailed Student's $t$ test.

production, we next tested whether STAT4 deficiency affects the bacterial killing capacity of neutrophils using MRSA (Figure 3E). As shown in Figure 3E, STAT4-deficient neutrophils demonstrated attenuated bacterial killing of MRSA in comparison with WT neutrophils. Phagocytosis is one of the major functions of neutrophils and the main defense mechanism against bacterial challenges. Interestingly, we found that phagocytosis of MRSA or E. coli (Supplemental Figure 2, C and D) by neutrophils was not altered in the absence of STAT4, suggesting that STAT4 modulates pathways that regulate many but not all neutrophil functions.

IL-12-dependent chemotaxis of human neutrophils. So far, our data demonstrated the functional activity of the IL-12 /STAT4 axis in murine neutrophils. Next, we tested the expression of IL-12R $\beta 1$ and IL-12ß2 by human peripheral blood neutrophils from healthy subjects using combined staining with anti-IL-12R $\beta 1$-FITC and IL-12ß2-Alexa Fluor 488 Abs. As shown in Figure 4A, human neutrophils expressed low but detectable levels of basal IL-12R $\beta 1 / \beta 2$ (combined staining with anti-12R $\beta 1$-FITC and IL-12 $\beta 2-A l e x a$ Fluor 488 is shown), which increased upon IL-12 treatment. IL-12 treatment also rapidly induced STAT4 phosphorylation in a time-dependent manner (Figure 4B). Human neutrophils not only expressed IL-12R, but they were also functionally responsive to IL-12. IL-12 increased human neutrophil chemotaxis to CXCL1 and GM-CSF in the Transwell assays, indicating that the IL-12/STAT4 axis is functional in human neutrophils (Figure 4C).

STAT4 is required for neutrophil extracellular trap formation. We next asked whether the IL-12/STAT4 axis is involved in the formation/release of neutrophil extracellular traps (NETs) (23). First, we examined elastase release upon neutrophil activation, which occurs as part of NET formation. In contrast to an induction of neutrophil elastase release from WT cells, neither LPS nor $P$. aeruginosa stimulated elastase release from Stat $4^{-1}$ and Stat $4^{l / f l} L y s M^{\text {cre }}$ neutrophils (Figure 5A). We then further examined features of NET formation by examining cell-associated but extracellular elastase and DNA presence by flow cytometry. While LPS, heat-killed 
A

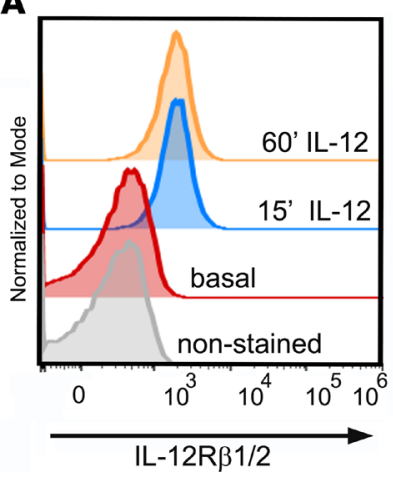

B

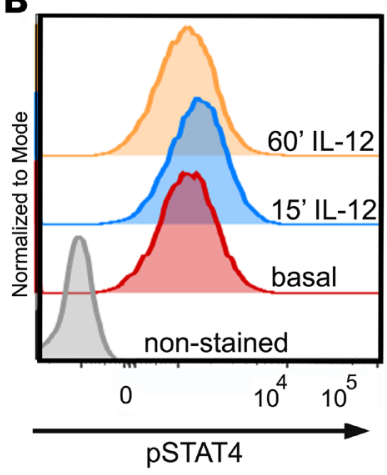

C

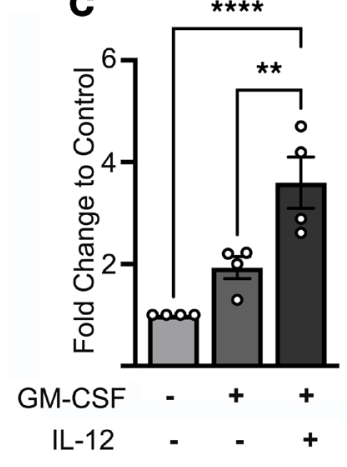

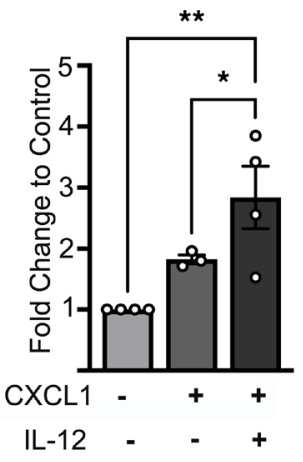

Figure 4. IL-12 induces STAT4 activation in human neutrophils and supports neutrophil chemotaxis in vitro. (A and B) Human neutrophils were incubated with IL-12 (40 ng/mL) for indicated time points; stained for p-STAT4-PE, IL-12R 31 (FITC), and IL-12Rß2 (Alexa Fluor 488); and analyzed by FACS. Representative histogram is shown (total: 4 donors in 2 independent experiments). (C) Isolated human neutrophils $\left(0.5 \times 10^{6} / 200 \mu \mathrm{L}\right.$ in RPMI 1640+1\% FBS) were either pretreated with IL-12 (40 ng/mL) or left untreated for 30 minutes and then seeded into Transwell inserts. Neutrophils migrated toward RPMI $1640+1 \%$ FBS media supplemented with $100 \mathrm{ng} / \mathrm{mL}$ recombinant human CXCL1 (rhCXCL1) or $50 \mathrm{ng} / \mathrm{mL}$ rGM-CSF, or migration media as a control, for 90 minutes at $37^{\circ} \mathrm{C}$ before being harvested and counted. The results depict neutrophil migration index normalized to the mean of the controls. ${ }^{*} P<0.05,{ }^{* *} P<0.01,{ }^{* * * *} P<$ 0.0001 using 1-way ANOVA followed by Tukey-Kramer post hoc test.

MRSA (HK-MRSA) and live MRSA, as well as Pam3CSK4 clearly induced surface-associated neutrophil elastase from WT neutrophils, STAT4 deficiency significantly attenuated surface staining of elastase (Figure 5 , $\mathrm{B}$ and $\mathrm{C}$ ). Importantly, the response to the Gram-positive bacteria MRSA and the Gram-negative HK-MRSA and MRSA $P$. aeruginosa or LPS was similarly defective in the absence of STAT4 (Figure 5, A-C). To directly test DNA release from the cells, we stained WT and STAT4-deficient neutrophils that were stimulated with live or HK-MRSA, Pam3CSK4, or LPS with Sytox Green, a cell-impermeable DNA dye. When stimulated with MRSA or Pam3CSK4, WT neutrophils showed significant externalization of DNA (Figure 5, D and E). In contrast, Stat $4^{--}$or Stat $4^{\text {fl/l }}$ Lys $M^{\text {rre }}$ neutrophils demonstrated reduced Sytox Green signal (Figure 5, D and E). Similar results were observed with $P$. aeruginosa stimulation (not shown). Thus, stimulation with both Gram-positive and Gram-negative bacteria induces STAT4-dependent externalization of DNA in neutrophils, suggesting a common mechanism of response in neutrophils that involves STAT4.

Next, we applied multispectral imaging flow cytometry to examine characteristics of neutrophils undergoing "suicidal" and "vital" NETosis (24). Cells under suicidal NETosis display features of a large nuclear area, nuclear decondensation, and colocalization of myeloperoxidase (MPO) with the DNA (24). Interestingly, we detected that Hoechst-stained DNA and MPO staining were separated in WT but had overlapping signals in Stat4 $4^{--}$neutrophils (Figure 6A). To quantify the colocalization of MPO and Hoechst staining, we calculated a Similarity Score (24) that reflects the degree of nuclear translocation of MPO. As shown in Figure 6B, the median Similarity Score for STAT4-deficient neutrophil was around +2.0 , indicating a positive correlation between the MPO and Hoechst/DNA images and therefore increased colocalization of MPO and Hoechst nuclear staining. Thus, cell compartmentalization was altered in Stat $4^{-1}$ neutrophils compared with control neutrophils as typically observed upon "suicidal" NETosis.

In contrast to "suicidal" NETosis, "vital" NETosis in neutrophils is characterized by multilobular/condensed nuclei, elongated cell shape with a polarized bleb, and minimal colocalization of MPO and nuclei (24). Upon detailed investigation of the shape and structure of WT and Stat4 ${ }^{-/-}$cells, we noticed that stimulated WT cells were elongated, a change that was absent in Stat $4^{-1-}$ neutrophils using BF microscopy images (Figure 6C). The aspect ratio, a measure of the spherical nature of the cell, was altered in WT but not Stat4 $4^{-1}$ cells (Figure 6C), suggesting that STAT4 deficiency reduces the signs of vital NETosis in neutrophils. We next

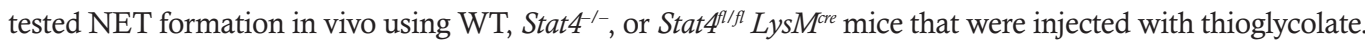
Based on the morphology/circularity parameters of cells, we analyzed 4 groups of neutrophils (Figure 6, D

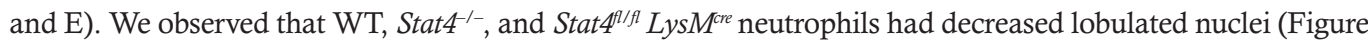

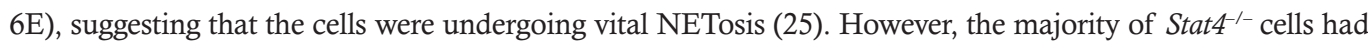
increased DNA condensation and diminished DNA release compared with WT cells (Figure 6E), suggesting impaired vital NETosis in the absence of STAT4.

STAT4-deficient mice are highly susceptible to MRSA infection. MRSA strains pose a significant public health threat in the community because they could overcome the neutrophil-mediated host defense to cause human 

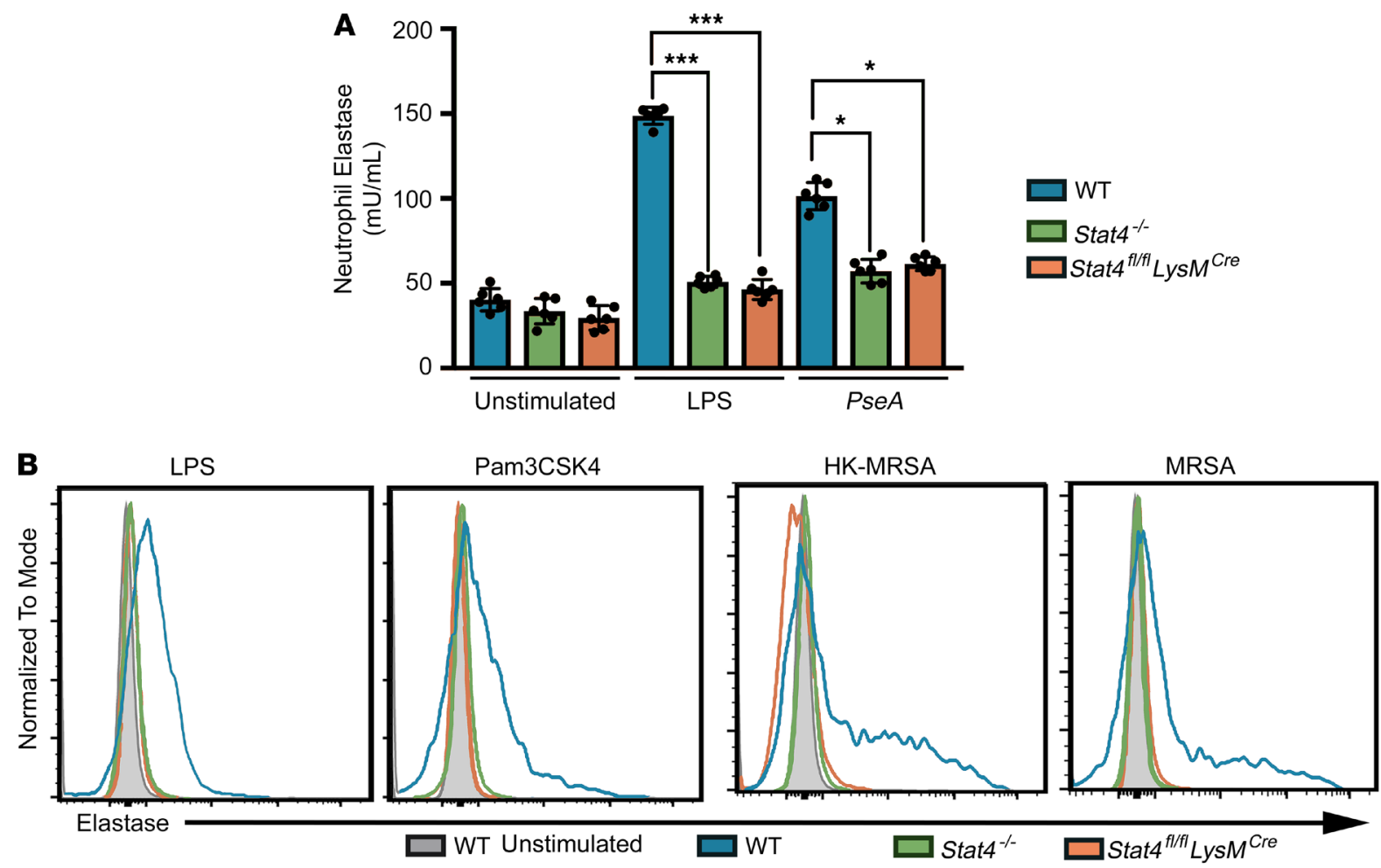

C

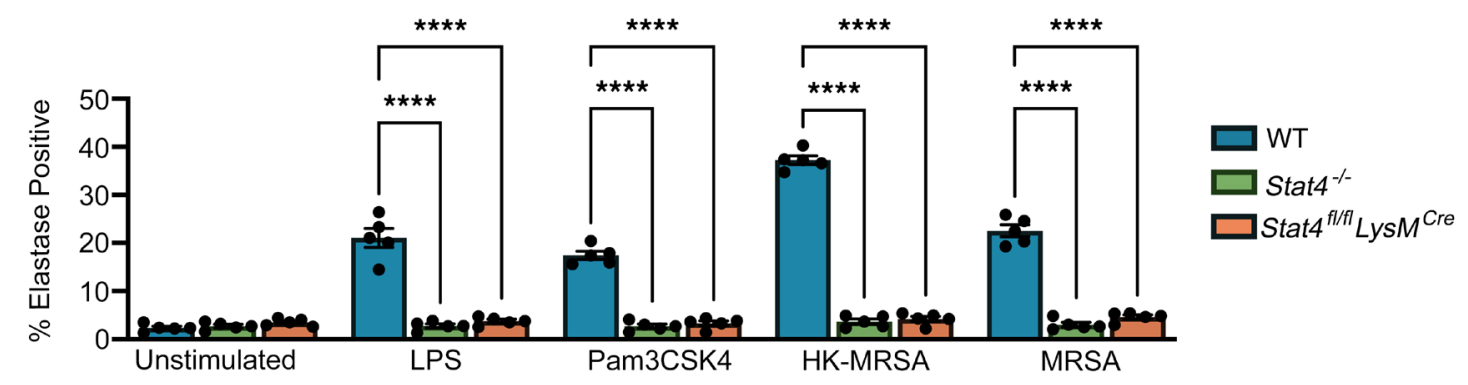

D

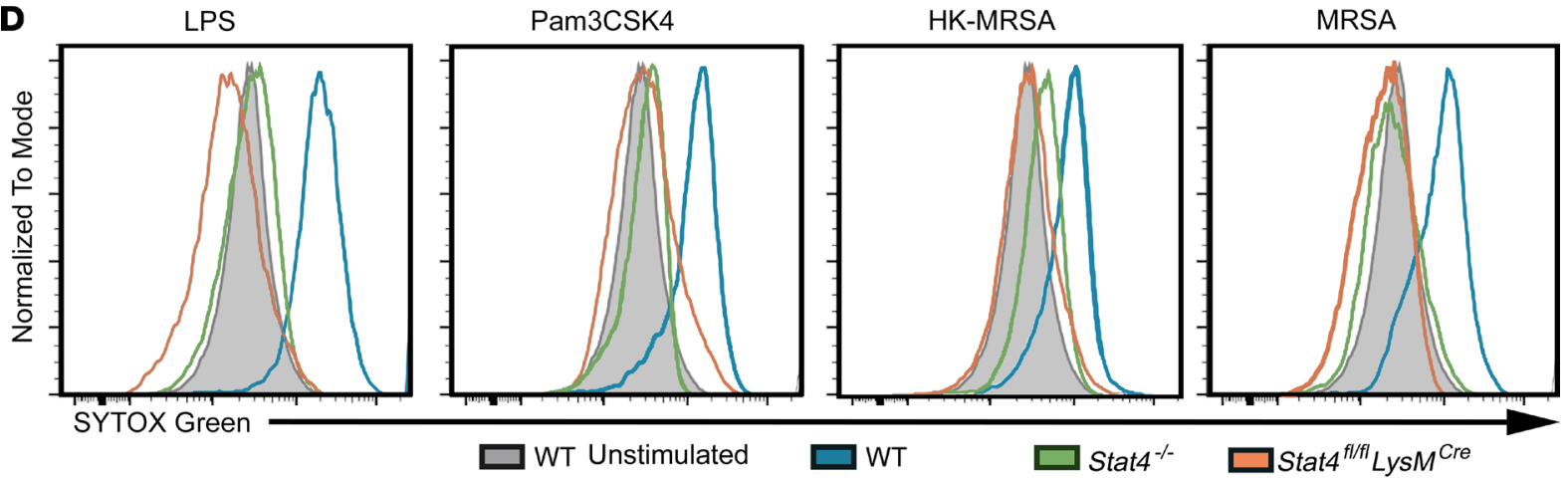

$\mathbf{E}$

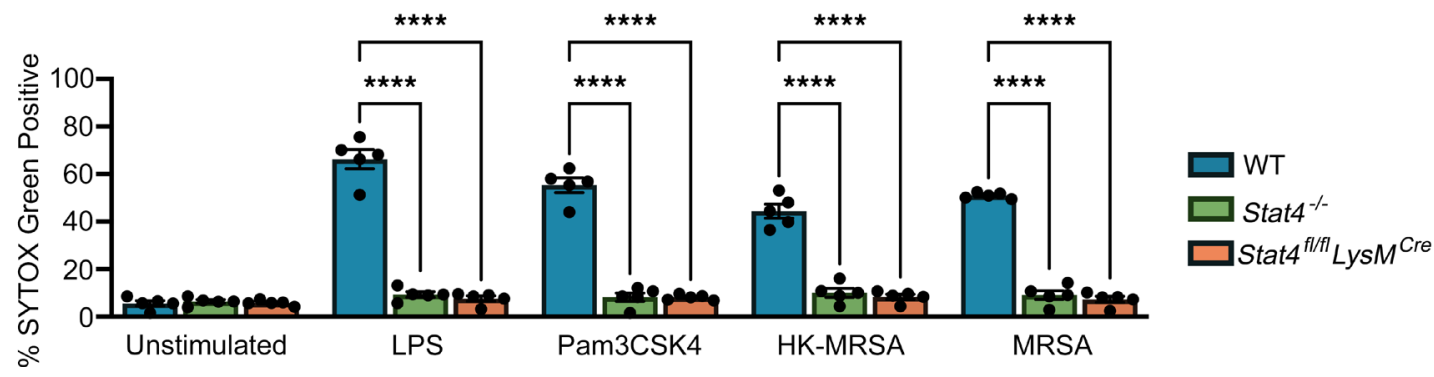


Figure 5. STAT4 supports TLR-dependent induction of elastase and DNA release from neutrophils. (A) Neutrophils from WT, Stat4 ${ }^{-/}$, and Stat4 ${ }^{f / f l}$ Lys $^{\mathrm{cre}}$ mice were stimulated with LPS or $P$. aeruginosa overnight. Supernatants were assayed for release of neutrophil elastase by ELISA ( $n=6$ mice in 2 independent experiments). (B) Purified neutrophils from BM cells from WT, Stat4 ${ }^{-/}$, and Stat $4^{\text {fl/f }}$ Lys $\mathrm{M}^{\text {cre }}$ mice were stimulated with LPS (100 ng/mL), Pam3CSK4 (10 ng/mL), HK-MRSA (10:1 MOI), or MRSA (10:1 MOI) for 60 minutes; stained for elastase (B and C) or Sytox Green ( $\mathbf{D}$ and $\mathbf{E}$ ); and examined by flow cytometry. (B) Representative histogram for elastase staining. (C) Percentage of elastase release from total Ly6C+CD11b+ neutrophils. (D) Representative histogram for Sytox Green staining. (E) Percentage of Sytox Green-positive out of total Ly6C + CD11b+ neutrophils. (C and E) ${ }^{*} P<0.05,{ }^{* * *} P<0.001,{ }^{* * * *} P<0.001$ using 1-way ANOVA followed by Tukey-Kramer post hoc test.

disease (26). Because we discovered an important role of the IL-12/STAT4 axis in neutrophil functions, we next asked whether STAT4 in myeloid cells, and particularly in neutrophils, is required for immunity during MRSA infection. First, we showed that MRSA reduced the survival of WT mice in a concentration-dependent

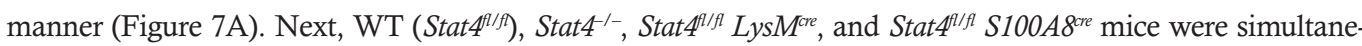
ously infected with MRSA, approximately $1 \times 10^{8} \mathrm{CFU}$, via i.p. injection. The global deletion of STAT4 resulted in increased bacterial burden and reduced survival (Figure 7, B and C). As with the experiment in global

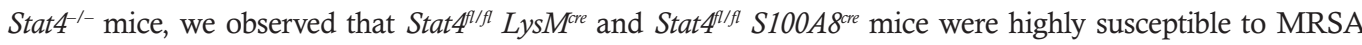
infection and demonstrated a substantial reduction in MRSA clearance and survival (Figure 7, B and C).

Mechanistically, MRSA infection resulted in a substantial recruitment of leukocytes to the PC, elevated percentage and number of $\mathrm{Ly}_{6} \mathrm{G}^{+}$neutrophils, and increased number of $\mathrm{F} 4 / 80^{+} \mathrm{Ly}_{6 \mathrm{C}}{ }^{+}$and $\mathrm{F} 4 / 80^{+} \mathrm{Ly} 6 \mathrm{C}^{-}$subsets of myeloid cells in WT (Stat4 ${ }^{f / f}$ ) mice (Figure 7, E and F). These changes were accompanied by $100 \%$

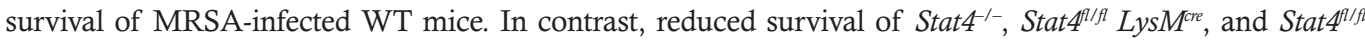
$S 100 A 8^{\text {cre }}$ mice was associated with diminished numbers of total peritoneal cells (Figure 7D). Interestingly, the total number of $\mathrm{F} 4 / 80^{+} \mathrm{Ly} 6 \mathrm{C}^{+} \mathrm{CD} 11 \mathrm{~b}^{+}$monocytes and $\mathrm{F} 4 / 80^{+} \mathrm{Ly} 6 \mathrm{C}^{-} \mathrm{CD} 11 \mathrm{~b}^{+}$macrophages were reduced

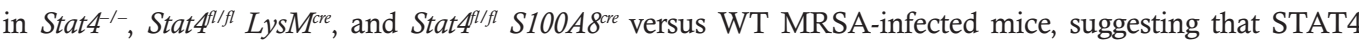
plays a role in the regulation of monocyte/macrophage recruitment upon MRSA infection (Figure 7, E-G). Surprisingly, STAT4 deficiency increased the relative percentage of $\mathrm{Ly}_{6} \mathrm{G}^{+} \mathrm{CD} 11 \mathrm{~b}^{+}$neutrophils in the PC of

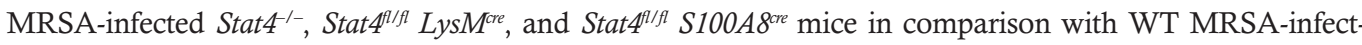
ed controls (Figure 7, E-G). However, the total number of neutrophils in the peritoneum was not different

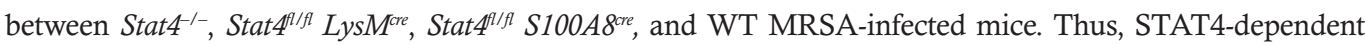
susceptibility to MRSA and inability to clear infection are likely due to defective neutrophil functions in the PC (ROS production, NETosis, bacterial killing) but not due to defective trafficking of neutrophils to the site of infection. Together, these data indicate that STAT4 is activated and required for many functions of neutrophils, thus playing a critical role in shaping antibacterial immunity.

\section{Discussion}

This study reveals a potentially novel role for STAT4 in the regulation of neutrophil functions. While STAT4 is a well-known transcription factor for Th1 cell differentiation, its expression and role in the regulation of neutrophil functions have not been previously identified. An increasing body of evidence suggests the implication of IL-12 and STAT4 in shaping of the innate immune responses in rodents and humans $(13-16,27)$, but cell types that are responsible for STAT4-dependent effects have not been well defined. Here, we demonstrate that STAT4 is expressed in neutrophils and activated by IL-12 and that activation results in elevated ROS production, increased signs of NETosis, and chemotaxis. Our finding is also of high interest as these data provide evidence for a critical role of STAT4 for neutrophil-dependent immunity against bacterial infections.

While there is a significant body of knowledge about transcriptional regulation of neutrophil differentiation and maturation, much less is known about signal transduction that regulates neutrophil functions. Neutrophils quickly respond to various stimuli via engagement of cell surface receptors, including Fc, adhesion, cytokine, and chemokine receptors, as well as innate immune receptors (17). These interactions lead to an induction of intracellular signal transduction pathways that direct and orchestrate critical neutrophil functions. For example, it has been demonstrated that STAT3 regulates CXCL2-induced migration via activation of the Raf/MEK/ ERK signaling axis (28). Interestingly, a role for IL-12 in myeloid cell functions became evident due to initial reports about effects of IL-12 on innate immune cells. Evidence indicates that IL-12R $\beta 1 / 2$ are expressed by human eosinophils at low or undetectable levels, and PMA activation dramatically induces IL-12R expression in these cells (29). IL-12R $\beta 1$ expression was also reported for human neutrophils (30). Here, we show that human neutrophils express both IL-12R $\beta 1$ and IL-12R $\beta 2$ and neutrophils are highly responsive to IL-12 stimulation in various functional assays via the activation of STAT4. As a part of an IL-12-dependent signaling cascade, IL-12 activates tyrosine kinase 2 (TYK2) and JAK2 to induce the phosphorylation of various STATs (5). 
A

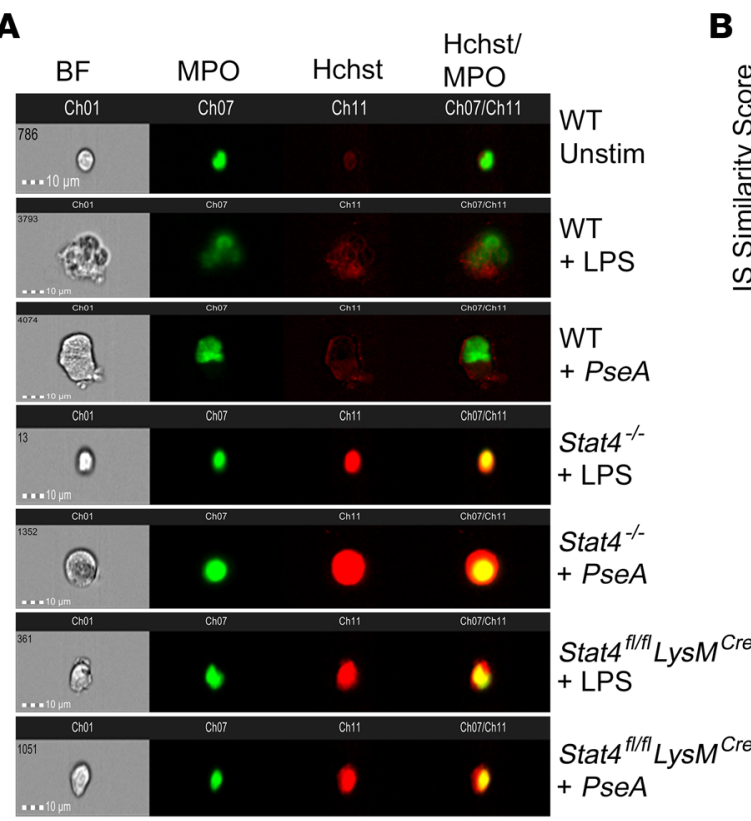

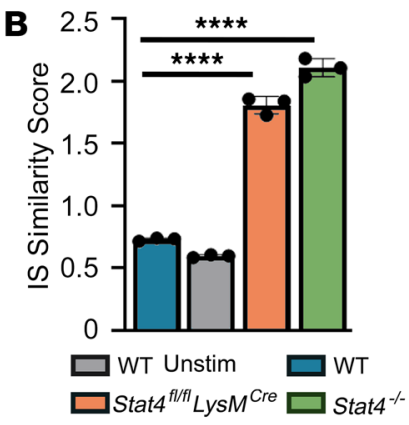

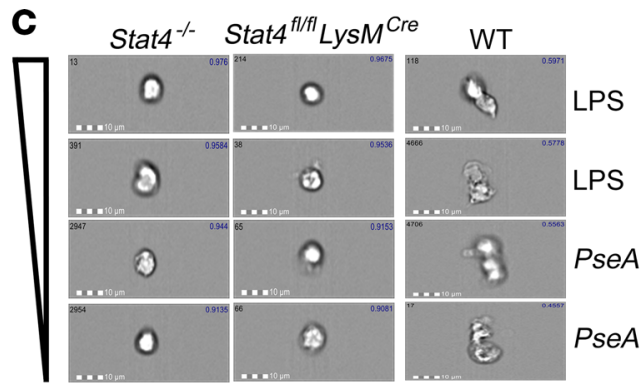

${ }^{\star *}$ Aspect Ratios=Minor Axis/Major Axis

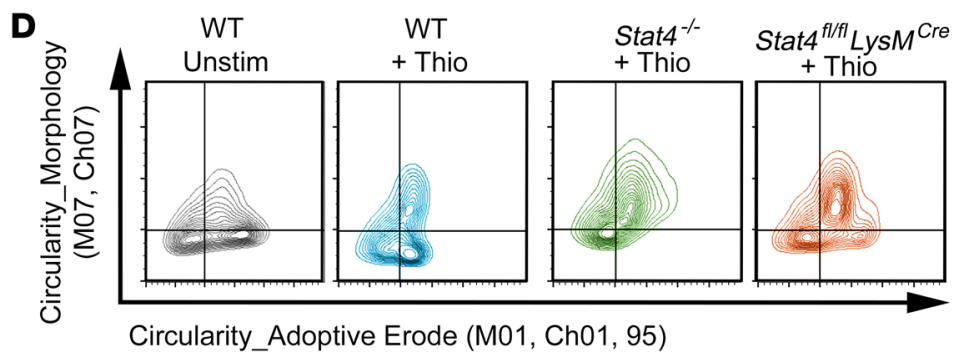

E

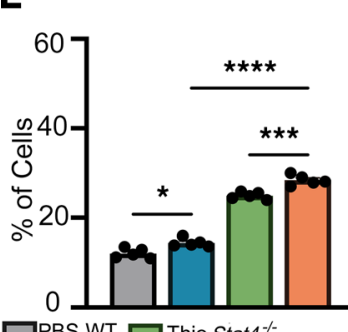

$\square$ PBS WT $\square$ Thio Stat4

$\square$ Thio WT $\square$ Thio Stat4 ${ }^{\text {flfil } L y S M^{\text {Cro }}}$

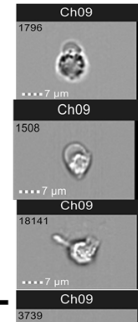

8

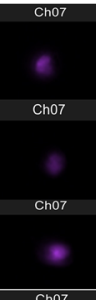

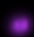

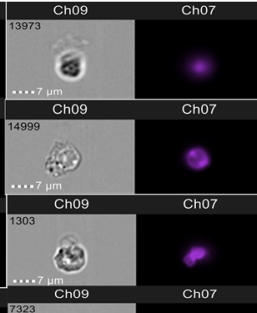

$\mathrm{s}$

(49.)
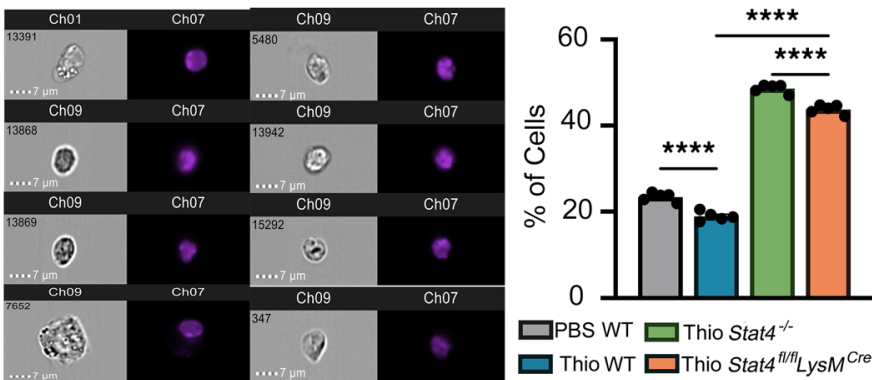

Membrane Blebbing

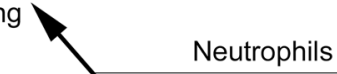

$\square$ PBS WT $\square$ Thio Stat4

$\square$ Thio WT $\square$ Thio Stat4 ${ }^{H / f l}{ }^{L} y s M^{\text {Cre }}$

DNA Decondensation

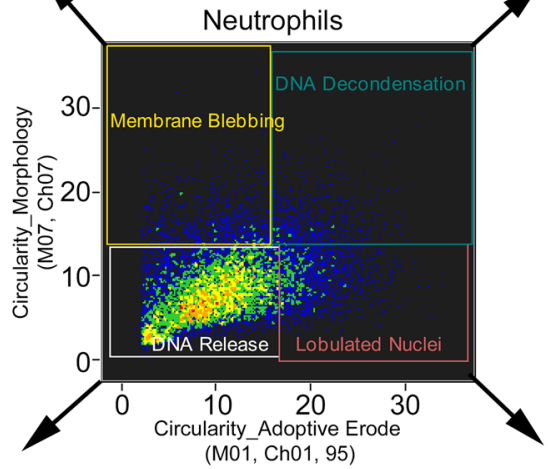

DNA Release (M01, Ch01, 95) Lobulated Nuclei

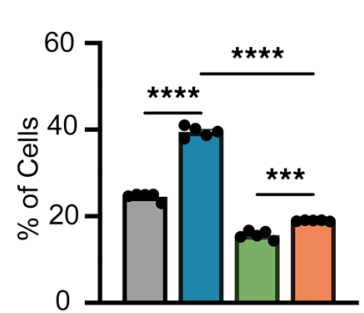

घPBS WT $\square$ Thio Stat4 ${ }^{-1}$

$\square$ Thio WT $\square$ Thio Stat ${ }^{\text {tffil }}$ LysM ${ }^{\text {Cre }}$
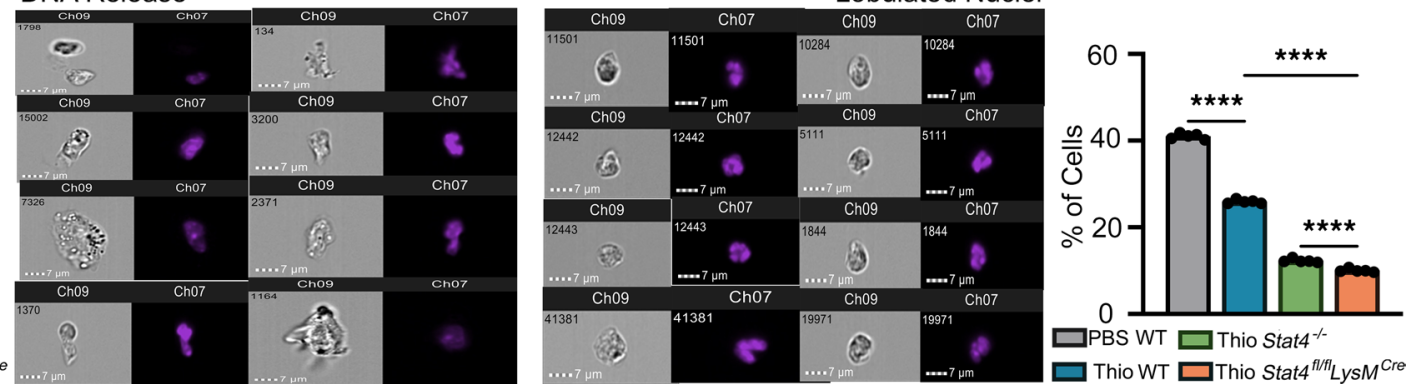
Figure 6. STAT4 alters multiple features of NET formation. BM neutrophils were stimulated with LPS (100 ng/mL) or P. aeruginosa (PseA; $10 \mathrm{MOI})$ and analyzed by ImageStream (IS) Multispectral Imaging Flow Cytometry. (A) Representative images of stimulated BM neutrophils in bright-field (BF), MPO, Hoechst (DNA staining), and overlay of MPO/Hoechst. (B) Colocalization of MPO and Hoechst staining (nuclear localization) calculated as a Similarity Score to quantify the degree of nuclear translocation of MPO; ${ }^{* * *} P<0.001$ using 2-way ANOVA with Tukey-Kramer post hoc test. Results are shown as the average of 50 cells for each of 3 mice in 2 independent experiments. (C) Morphology changes following LPS and P. aeruginosa treatment in WT, Stat4 ${ }^{-/-}$, and Stat $4^{f / f l}$ LysM ${ }^{c r e}$ neutrophils. (D and E) Singlet gated neutrophils were analyzed for nuclear circularity versus cell circularity. The images shown were obtained from events in each quadrant. (D)

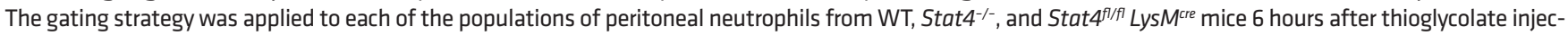
tion. (E) Representative FACS plots and the percentage of the PC neutrophils in different stages of the NETs' formation. ${ }^{*} P<0.05,{ }^{* * *} P<0.001,{ }^{* * * *} P<0.0001$.

JAK2 activation is required for IL-12-mediated T cell growth, whereas the TYK2/STAT4 signaling pathway is critical for IFN production. To date, little is known about a transcriptional network that regulates neutrophil functions. In this report, we demonstrated that JAK2 inhibition attenuated STAT4 phosphorylation, indicating that IL-12 activates the JAK2/STAT4 pathway in neutrophils. STATs are well-known for many functions in innate immunity. STAT1 supports IFN-dependent responses, STAT3 is downstream of IL-6, and cytokines such as colony-stimulating factors also signal via various STATs (31). We now add STAT4 to a list of transcription factors that play a crucial role in shaping of neutrophil functions.

Our data demonstrate that STAT4 deficiency has no effects on peripheral number of neutrophils under homeostatic conditions but modulates gene expression in neutrophils at the basal and activated levels. These results suggest that STAT4 activation in neutrophils is an integral component of inflammatory immunity, at least in the context of a bacterial infection. In this report, we have shown that stimulation with IL-12 activates STAT4. STAT4 might also be activated by type I IFNs and IL-23 (32), and either of those cytokines might be additional stimulators of STAT4-dependent neutrophil activity. To further explore consequences of STAT4 activation in neutrophils, we focused our attention on antimicrobial responses in neutrophils. Interestingly, we found that STAT4, at least partially, suppresses granule release from neutrophils at the basal conditions but supports activation of inflammasome and NF- $\mathrm{BB}$ upon the activation with IL-12. We also found that IL-12 has some STAT4-independent effects that likely work via other STATs, such as STAT1, STAT3, and STAT5. Overall, gene expression data clearly demonstrate that the IL-12/STAT4 axis actively participates in shaping neutrophil biology at homeostasis and under inflammatory conditions.

In terms of functional consequences of IL-12/STAT4-dependent activation, we first investigated ROS production by IL-12-activated neutrophils. Our data revealed that IL-12 induces oxidative burst in neutrophils and this process is STAT4 dependent. Cytokines use various signaling cascades to activate ROS production in neutrophils (33). TNF- $\alpha$ induces p38 MAPK-dependent mobilization of cytb558 to the plasma membrane (34). Priming of neutrophils by GM-CSF leads to the phosphorylation of p47phox and the translocation and docking of the cytosolic complex to the membrane (35). At present, the molecular basis for STAT4-dependent oxidative burst in neutrophils is not well understood, and future experiments should be focused on defining the mechanism underlying STAT4 effects in control of ROS production in neutrophils. The generation of ROS plays an important role in the antimicrobial functions of the phagocytic cells, and ROS supports phagocytosis and intracellular bacteria killing. Killing of $S$. aureus by neutrophils in vitro is highly dependent on NADPH oxidase activity (36), and our data now highlight the importance of STAT4 in this process. Importantly, STAT4 also regulates bacterial killing of MRSA that is highly associated with ROS synthesis. Phagocytosis and ROS generation are major cellular mechanisms of the microbial clearance, but their interplay is not well understood. While the main mechanism of phagocytosis is also accompanied by ROS production, recent evidence suggests that ROS-independent phagocytosis might exist (37). Our data, in line with this report, demonstrate that STAT4-dependent ROS synthesis by neutrophils has a minimal impact on successful phagocytosis of MRSA. Overall, our data indicate that effects of STAT4 are specific to certain but not all neutrophil functions.

IL-12 is induced by various types of pathogens, including bacteria, protozoa, helminths, fungi, and viruses (38). Dendritic cells, monocytes, macrophages, and to a lesser extent B cells are the source of IL-12. Additionally, microglia, infected keratinocytes and osteoblasts, and epithelial and endothelial cells serve as a source of IL-12. Neutrophils stimulated by LPS also release IL-12, and IFN- $\gamma$ enhances the LPS-induced secretion of IL-12 (39). Interestingly, IL-12 is found in granules of neutrophils and is quickly released upon stimulation, suggesting that neutrophils have a positive feedback loop in their activation via IL-12. As IL-12 induces neutrophil activation and induction of the Th1 response via the induction of IFN- $\gamma, \mathrm{IL}-12$-producing neutrophils play an active role in the regulation of innate and adaptive responses. Interestingly, we found that TLR engagement induces ROS generation in a STAT4-dependent manner. Several studies have reported that neutrophils have IL-12-containing granules that can be released upon neutrophil activation. Our data suggest that TLR-induced 
A

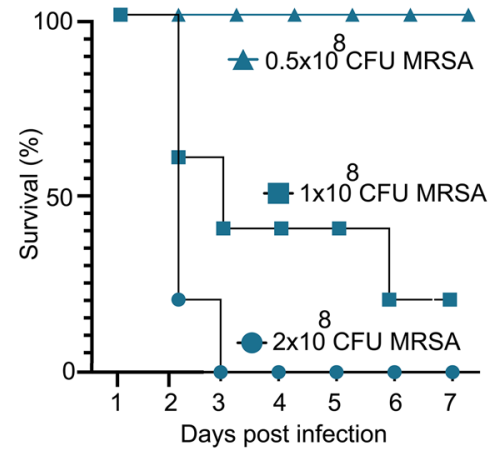

B

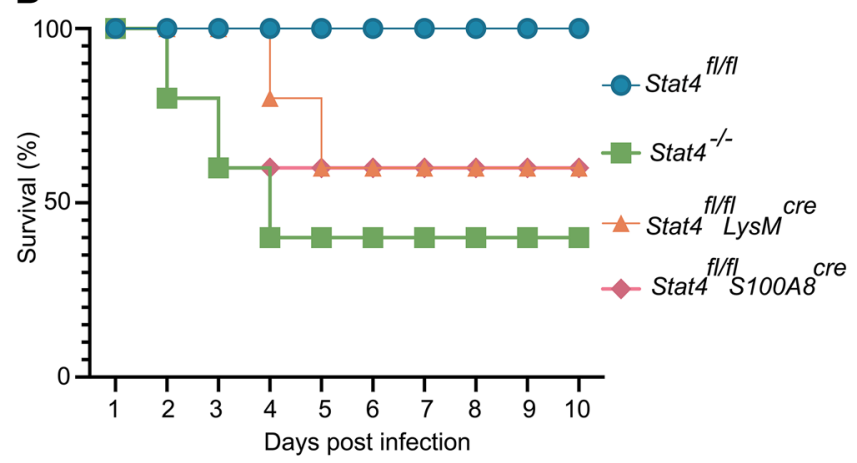

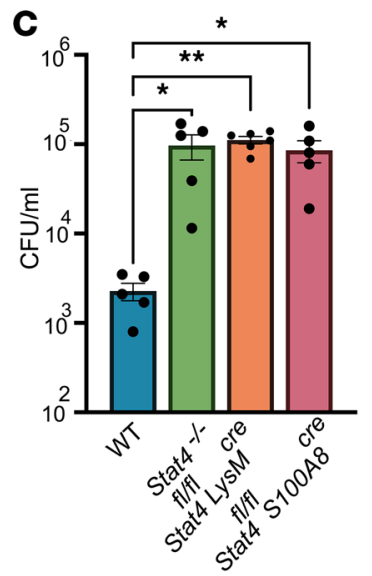

+MRSA
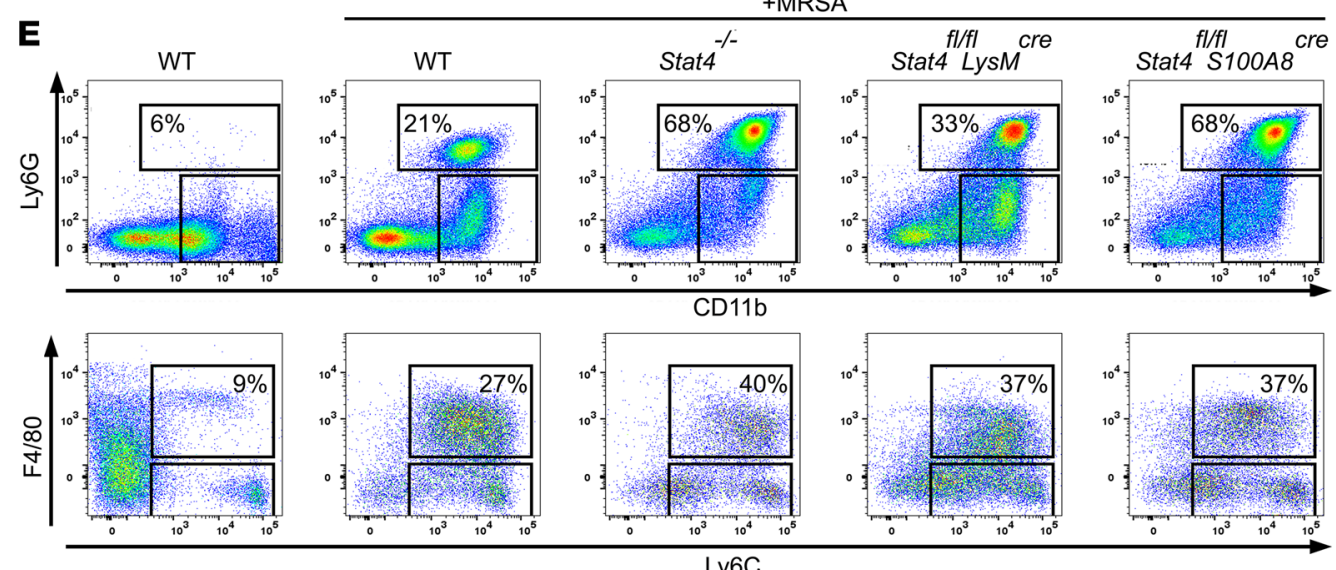

$\%$ of Live Singlets
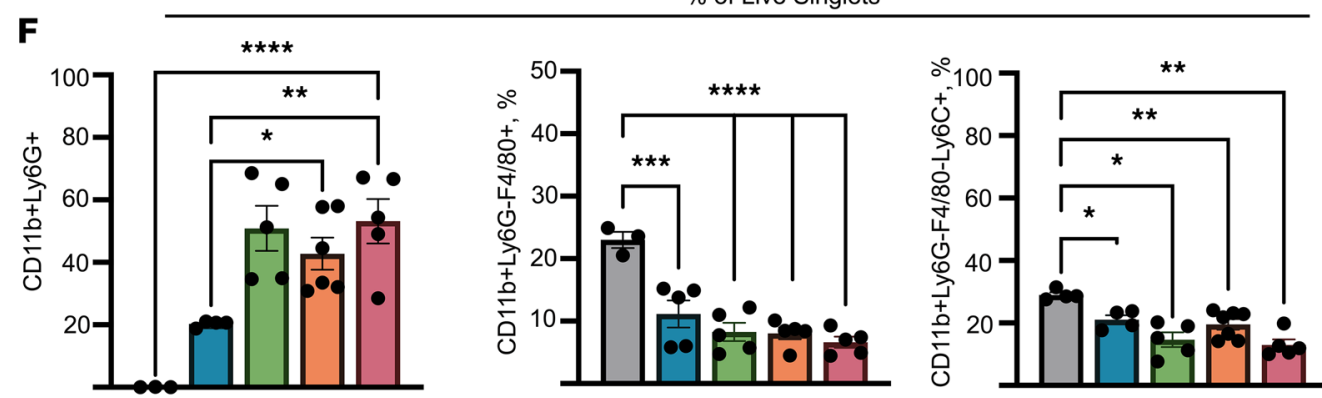

D

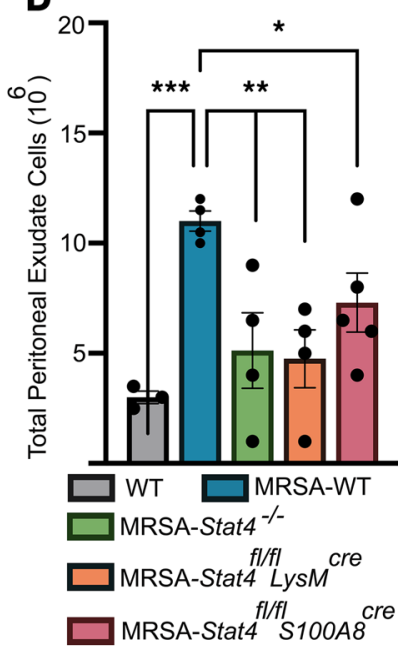

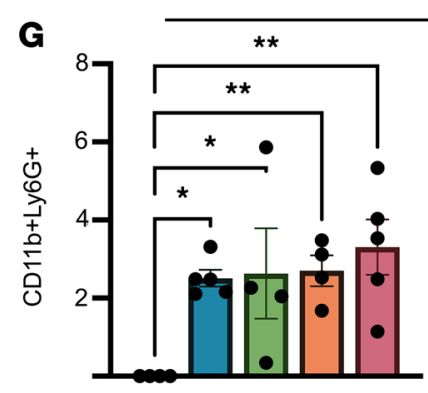

Number of cells $\left(x 10^{6}\right)$
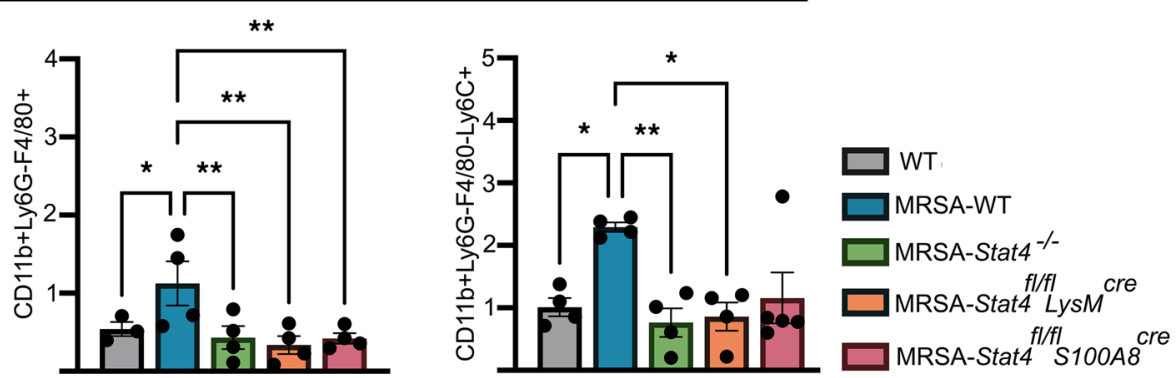

Figure 7. STAT4 is required for immunity to MRSA peritoneal infection. (A) WT mice were infected with different concentrations of MRSA. Survival rates are presented ( $n=5-7 /$ group). (B) WT (Stat $\left.4^{f / f l}\right)$, Stat4 ${ }^{-/-}$, Stat $4^{f / f l}$ Lys $^{\text {cre }}$, and Stat4 ${ }^{f / f l}$ S100A $8^{\text {cre }}$ mice were infected with MRSA (1 $\left.\times 10^{8}\right)$ i.p. Survival rates are presented ( $n=10$ mice/group). (C) CFU counts in the PC of WT, Stat4 ${ }^{-1-}$, Stat4 ${ }^{f / f l}$ Lys $M^{\text {cre }}$, and Stat4 fl/fl S100A $8^{\text {cre }}$ infected mice 24 hours after MRSA infection ( $n=5 /$ group).

(D) Total number of peritoneal exudate cells collected from WT, Stat4 ${ }^{-/-}$, Stat $4^{\text {fl/fl }}$ LysM $^{\text {cre }}$, and Stat4 ${ }^{\text {flfl }}$ S100A8 $8^{\text {cre }}$ mice ( $n=4 /$ group). (E) Top: Representative FACS

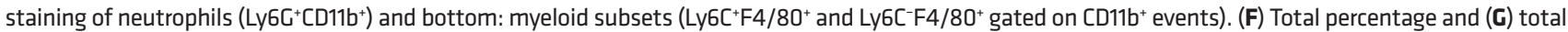

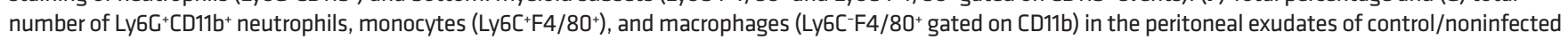
and MRSA-infected WT, Stat4 ${ }^{-/-}$, Stat ${ }^{f / f l}$ LysM ${ }^{\text {cre }}$, and Stat $4^{\text {fl/fl }}$ S100A $8^{\text {cre }}$ mice $\left(n=4 /\right.$ group). ${ }^{*} P<0.05$, ${ }^{* *} P<0.01$, ${ }^{* * *} P<0.001,{ }^{* * *} P<0.0001$, using 1-way ANOVA followed by Tukey-Kramer post hoc test. 
activation initiates IL-12 release and further supports IL-12-induced activation of neutrophils that depends on STAT4. It remains to be determined whether it could be a general mechanism for support of neutrophil functions such as bacterial killing and NETosis.

The finding that STAT4 is essential for NET formation is surprising and suggests additional STAT4-dependent immune functions of neutrophils in various disease settings. Gram-positive MRSA, an agonist to TLR2; Gram-negative bacteria P. aeruginosa; and the TLR4 agonist LPS induce elastase and extracellular DNA release that are both signs of NETosis. NETs have been shown to be important for autoimmune diseases (40), and considering the genetic link between STAT4 and various autoimmune diseases in human populations (7), it is possible that some of the impact of STAT4 on human disease is through activity in innate immune cells such as neutrophils. The details of this contribution will be important to define in future studies. NETosis is observed in 2 major forms, suicidal and vital NETosis (25). In this report, we mainly focused on the morphological changes supporting NETosis and demonstrated that STAT4 inhibits suicidal NETosis but supports steps for vital NETosis, such as changes in cell shape and DNA release. It remains to be determined how STAT4 regulates NETosis and whether activation of NADPH oxidase 2 is a necessary requirement for this process.

One of the key properties of neutrophils is an ability to migrate to a site of inflammation. Our data showed that STAT4 deficiency already diminishes chemotaxis toward CXCL1 and GM-CSF in the in vitro assays. The simplest explanation for this observation would be that STAT4 deficiency likely affects cell-intrinsic properties of neutrophils, such as motility, polarity, integrin-dependent cytoskeleton organization, or expression of chemokine receptors, and further studies should reveal a detailed mechanism for STAT4-dependent neutrophil migration.

One of the key and definitive findings of this study was demonstrating that STAT4 plays a role in antibacterial immunity via the control of MRSA bacterial load. Here, we demonstrated that global, myeloid, and neutrophil-specific STAT4 deficiency substantially reduced survival of mice after MRSA infection. Interestingly, these data also highlighted an independent role of myeloid cells, and particularly neutrophils, in this process. While peritoneal MRSA infection led to leukocyte recruitment to the peritoneum, STAT4

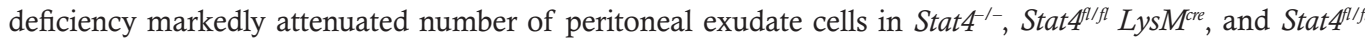
S100A $8^{\text {re }}$ mice. In contrast to the observed defective migration in the in vitro assays, STAT4-deficient and WT neutrophils migrated equally well to the site of infection. The detected differences between the in vitro Transwell assays and in vivo observed phenotypes might be due to a more complex milieu of signals that are involved in neutrophil recruitment in vivo. Peritoneal cell exudate from WT-infected mice also contained $\mathrm{F} 4 / 80^{+} \mathrm{Ly} 6 \mathrm{C}^{+}$monocytes and $\mathrm{F} 4 / 80^{+} \mathrm{Ly} 6 \mathrm{C}^{-}$macrophages, and these populations were reduced in

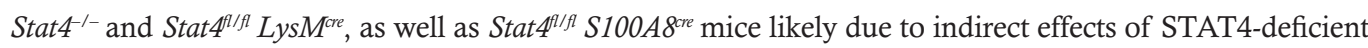
neutrophils on monocyte recruitment to the peritoneum at least in the case of Stat $4^{1 / f l} S 100 A 8^{\text {cre }}$ mice. If the numbers of neutrophils are similar in the peritoneum between STAT4-deficient and WT mice, why do

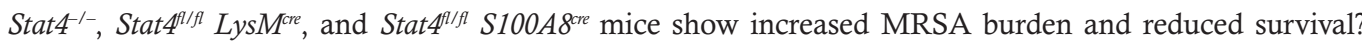
Neutrophil binding and ingestion of invading $S$. aureus trigger potent oxidative and nonoxidative antimicrobial killing mechanisms (41). We believe while STAT4-deficient neutrophils were able to migrate to the infected peritoneum, their flawed capacity to produce ROS and NETs and perform bacterial killing was a critical component that is lacking in the successful elimination of MRSA infection.

In summary, in this report, we described a requirement for STAT4 in regulation of several key neutrophil functions and demonstrated that STAT4 plays a critical role in antimicrobial immunity. We have used a newly generated myeloid cell-specific and neutrophil-specific STAT4-deficient mouse model that allowed us to exclude any additional effects from other STAT4-expressing cells. The study reported here might also raise some questions about interpretations of previous work involving STAT4. While much of the function of STAT4 has been attributed to T cells, the results from in vivo studies using germline Stat $4^{-{ }^{-}}$mice could be reexamined from the perspective that STAT4 could contribute to the activity of innate immune cells that might also affect disease phenotypes. Defining exactly how the IL-12/STAT4 axis controls neutrophil activation should be an interesting and important area of further investigation.

\section{Methods}

Mice. The study was performed with C57BL/6J (WT) (The Jackson Laboratory, JAX: 000664) mice, mice that

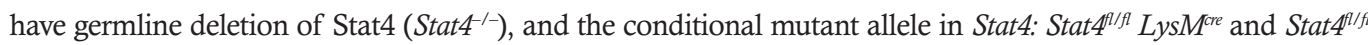
$S 100 A 8^{\text {rer }}$ mice. Stat $4^{l / f l}$ mice were generated by Ozgene Inc. (Australia), and breeding pairs were shipped to Eastern Virginia Medical School and Indiana University to generate Stat $4^{l / f l}$ LysM ${ }^{\text {rre }}$ and Stat $4^{\text {Ilfl }}$ S100A $8^{\text {rre }}$ mice. Breeding pairs of $L y s M^{c r e}$ and S100A8 gre (JAX: 004781 and JAX: 021614, respectively) were purchased from JAX. 
All mice were on C57BL/ 6 background. Mice were generated with the third exon in the Stat 4 gene being flanked with loxP sites and containing an FRT-flanked neogene. Correctly targeted pups were identified by Southern blot and crossed to Flp-transgenic mice to delete the neogene. Neodeleted Stat $4^{+/ f t}$ mice were crossed with the specified Cre-transgenic mice to mediate deletion of exon 3 in a lineage-specific manner. This approach was chosen since splicing of the remaining exons (with the ATG start codon in exon 2) will result in a nonsense transcript arising from a frameshift during translation.

Western blotting. BM neutrophils from WT, Stat $4^{1 / f l} L y s M^{\text {cre }}$, and Stat $4^{\text {Ilfl }}$ S100A gore mice were isolated via negative selection kit (STEMCELL Technologies, 19762A) and stimulated with $40 \mathrm{ng} / \mathrm{mL}$ IL-12 (Peprotech, 210-12) in RPMI with 10\% FBS for the indicated time points. Following stimulation, cells were washed and lysed with lysis buffer (Thermo Fisher Scientific, 78501) contacting protease inhibitor (Thermo Fisher Scientific, 78429) and phosphatase inhibitor cocktails (Cell Signaling Technology, 5870S). Protein content was determined by BCA method (Thermo Fisher Scientific), and $20 \mu \mathrm{g}$ of protein lysate was resolved on a 4\%-20\% polyacrylamide gradient gel, followed by transfer to PVDF membrane via semidry transfer. Membranes were blocked at room temperature for 1 hour with TBS-based blocking buffer (LI-COR Biosciences 927-50000). The following primary Abs were incubated at $4^{\circ} \mathrm{C}$ overnight: STAT4 (Cell Signaling Technology C46B10), p-STAT4 (BD Biosciences 612739), JAK2 (Cell Signaling Technology 3230), p-JAK2 (Cell Signaling Technology, 3771), and GAPDH (Santa Cruz Biotechnology, 32233). Secondary Abs (926-68072 and 926-32211, Li-COR Biosciences) were incubated at room temperature for 1 hour. Images were acquired using the LI-COR Odyssey system. Band intensity was determined using Image Studio Lite software (LI-COR Biosciences).

Antimicrobial pathway PCR arrays. Isolated peripheral blood neutrophils $\left(2 \times 10^{6}\right)$ from WT and Stat ${ }^{-1-}$ mice were incubated with or without IL-12 (100 ng/mL) for 3 hours in complete RPMI-1640 medium (Gibco, Thermo Fisher Scientific). Total RNA from all groups was extracted using RNeasy Mini Kit (QIAGEN, 74106). The concentration and purity of the RNA were determined using a NanoDrop 8000 spectrophotometer (Thermo Fisher Scientific). To eliminate the possible amplification of contaminating genomic DNA, DNase treatment (QIAGEN) was carried out, and total RNA $(1 \mu \mathrm{g})$ was reverse-transcribed to cDNA using the RT2 First Strand Kit (QIAGEN, 330404). Mouse RT² Profiler PCR Array Mouse Antibacterial Response (PAMM-148Z, QIAGEN) was used for the analysis. A negative control for genomic DNA and contaminating RNA was also conducted in each sample. Amplification, data acquisition, and the melting curve were carried out by the Real Time PCR system (Applied Biosystems, Thermo Fisher Scientific). The Ct and melting curve of each gene were automatically established and recorded by the software. The $\Delta \mathrm{Ct}$ method was used for PCR array data analysis. The normalized $\Delta \mathrm{Ct}$ for each gene of interest (GOI) was calculated by deducting the average $\mathrm{Ct}$ of the 5 housekeeping genes from the $\mathrm{Ct}$ of each GOI. Then the double delta $\mathrm{Ct}(\Delta \Delta \mathrm{Ct})$ for each GOI was calculated by deducting the average $\Delta \mathrm{Ct}$ of GOI in the sham group from the $\Delta \mathrm{Ct}$ of each GOI. The fold change of each GOI compared with the sham group was calculated as $2^{-\Delta \Delta C t}$.

Murine and human neutrophil isolation. BM neutrophils were isolated from the PBS-flushed femur and tibia bones of BM of 6- to 12-week-old mice. Obtained cell suspension was washed, then resuspended at $1 \times 10^{8}$ cells $/ \mathrm{mL}$ in PBS $+0.5 \%$ BSA and $2 \mathrm{mM}$ EDTA. Murine neutrophils were isolated from the peripheral blood or BM using EasySep Mouse Neutrophil Enrichment Kit (STEMCELL Technologies), and human neutrophils were isolated from peripheral blood of healthy donors at Eastern Virginia Medical School using EasySep Direct Neutrophil Isolation Kit (STEMCELL Technologies).

Transwell migration assays. BM neutrophils $\left(0.25 \times 10^{6}\right)$ from WT, Stat $4^{-/}$, and Stat $4^{\text {Ilfl }}$ Lys $^{\text {cre }}$ mice were suspended in $0.1 \mathrm{~mL}$ RPMI 1640 supplemented with 1\% FBS and $10 \mathrm{mM}$ HEPES ("migration media" hereafter) and seeded onto the top well of the Transwell. The bottom wells were loaded with $0.6 \mathrm{~mL}$ migration media supplemented with the following: media alone, $12-100 \mathrm{ng} / \mathrm{mL} \mathrm{rmCXCL} 1$, or $50 \mathrm{ng} / \mathrm{mL} \mathrm{rGM}-\mathrm{CSF}$ (both Peprotech). The loaded cells were allowed to migrate for 3 hours at $37^{\circ} \mathrm{C}$ before being harvested and counted. The migration index indicates the percentage of transmigrated neutrophils normalized to the mean of the controls for each genotype.

Isolated human peripheral blood neutrophils were resuspended in migration media and incubated for 30 minutes with IL-12 (40 ng/mL) before loading into Transwell inserts (3.0 $\mu \mathrm{m}$ pore, Corning). The top wells of the Transwell chambers were loaded with $0.5 \times 10^{6}$ neutrophils in $200 \mu \mathrm{L}$ of migration media. The bottom wells were loaded with $0.6 \mathrm{~mL}$ migration media either alone or supplemented with $100 \mathrm{ng} / \mathrm{mL}$ rhCXCL1 or 50 $\mathrm{ng} / \mathrm{mL}$ rGM-CSF (both Peprotech). Neutrophils were allowed to migrate for 90 minutes at $37^{\circ} \mathrm{C}$ before being harvested and counted. The migration index indicates the percentage of transmigrated neutrophils normalized to the mean of the controls for each condition. 
CXCL1 and GM-CSF-induced recruitment of neutrophils. WT, Stat $4^{-1-}$, and Stat ${ }^{\text {flfl }}$ LysM $^{\text {rre }}$ mice were injected i.p. with either $300 \mathrm{ng}$ rmCXCL1, $300 \mathrm{ng}$ rmGM-CSF, or $0.9 \% \mathrm{NaCl}$ for negative control. After 4 hours, mice were sacrificed; peritoneal lavage was collected, stained for $\mathrm{CD} 11 \mathrm{~b}^{+} \mathrm{Gr}-1^{+}$cells, and analyzed by FACS.

Flow cytometry. Single-cell suspensions from the BM, peritoneal cell exudate, and heparinized peripheral blood were stained with Abs as we previously described (42) and analyzed using a Cytek DXP8 Color (Cytek Development Inc.) with upgraded FACSCalibur (BD Biosciences) for ROS production, p-STAT4, IL-12R $\beta 1 / 2$, and purity check staining and Attune NxT flow cytometer (ABI) for elastase and Sytox Green staining. Data were analyzed using FlowJo (Tree Star Inc.). For all flow cytometry experiments, gates were set based on isotype and fluorescence minus one controls. The anti-mouse Abs used were as follows: Ly6C-APC (HK1.4), CD45-PerCP (30-F11), CD11b-Pacific Blue (M1/70.15), Ly6G-FITC (1A8), F4/80-eFluor450 (BM8.1), and anti-mouse CD16/CD32 Abs (Lymphocyte Culture Center, University of Virginia Health System) for blocking. For FACS on human neutrophils, $100 \mu \mathrm{L}$ whole blood was stimulated with $40 \mathrm{ng} / \mathrm{mL}$ rhIL-12 for 0 , 15, or 60 minutes. Following IL-12 stimulation, cells were surface stained with anti-CD15-Pacific Blue (clone MMA, BioLegend), anti-CD16-PerCPCy5.5 (clone 3G8, BioLegend), and anti-IL-12Rß2-Alexa Fluor 488 (clone 305719, R\&D Systems, Bio-Techne) or anti-IL-12Rß1-FITC (clone 69310, R\&D Systems, Bio-Techne) for 30 minutes at $4{ }^{\circ} \mathrm{C}$. Following surface staining, cells were fixed and permeabilized for intracellular p-STAT4 detection using the True-Nuclear Transcription Factor Buffer Set (BioLegend), following the manufacturer's protocol. Cells were incubated with anti-p-STAT4-PE Abs (clone 4LURPIE, BioLegend) for 30 minutes at $4^{\circ} \mathrm{C}$. To distinguish between live and dead cells in flow cytometry experiments, a LIVE/DEAD Aqua Dead Cell Stain Kit (Invitrogen, Thermo Fisher Scientific) was used.

Detection of ROS. BM was harvested from 6- to 10-week-old WT, Stat $4^{\text {flfl }}$ Lys $M^{\text {cre }}$, or Stat $4^{\text {flfl }}$ S100A8cre mice by flushing the femur and tibia bones with cold PBS. Cells were centrifuged at $300 \mathrm{~g}$ for 7 minutes and resuspended in $500 \mu \mathrm{L}$ advanced RPMI (Gibco, Thermo Fisher Scientific). Neutrophils were stained with $0.2 \mu \mathrm{M}$ DCFDA (Thermo Fisher Scientific, D-399) for 30 minutes at $37^{\circ} \mathrm{C}$ in the dark. Next, cells were incubated with either $40 \mathrm{ng} / \mathrm{mL}$ IL-12 (Peprotech, 210-12) or LPS (100 ng/mL) in advanced RPMI at $37^{\circ} \mathrm{C}$ with $5 \% \mathrm{CO}_{2}$. In some experiments for LPS-induced ROS production, neutrophils were pretreated with IL-12 neutralizing $\mathrm{Ab}$ (C17.8, BioLegend). After 1 or 14 hours, neutrophils were collected, washed with $1 \times$ wash buffer, stained with anti-CD11b and anti-Ly6G Abs, and analyzed by FACS.

Apoptosis. BM was harvested from 6- to 10-week-old WT mice and incubated with $40 \mathrm{ng} / \mathrm{mL} \mathrm{IL}-12$ (Peprotech, 210-12) for 30 minutes, 2 hours, 6 hours, and 12 hours at $37^{\circ} \mathrm{C}$ with $5 \% \mathrm{CO}_{2}$. Following IL-12 incubation, $1 \times 10^{6}$ cells were washed twice with ice-cold PBS, resuspended with $1 \mathrm{~mL}$ of binding buffer $(0.01$ M HEPES at pH 7.4, 0.14 M NaCl, $2.5 \mathrm{mM} \mathrm{CaCl}_{2}$ ), stained with annexin V-APC and 7-amino-actinomycin $\mathrm{D}$ (BD Pharmingen, 550475 and 559925 , respectively) for 15 minutes at $25^{\circ} \mathrm{C}$ in the dark, washed, and analyzed by FACS within 1 hour.

Phagocytosis and killing assays. Bacterial phagocytosis and killing were performed as previously described (43) Briefly, WT or STAT4-deficient neutrophils $\left(2 \times 10^{5}\right.$ /well) were plated into 2 individual 96-well plates with opaque walls and clear bottoms. Cells were pretreated with IL-12 for 1 hour before the addition of GFP-MRSA at a multiplicity of infection of 50:1. Infected cells were incubated 1 hour to allow phagocytosis, both plates were washed with warm PBS, and GFP fluorescence was measured on the first plate. The second plate was then maintained in PBS with or without IL-12 and was incubated for another 2 hours for killing assays. To measure the intensity of intracellular GFP fluorescence, extracellular fluorescence was quenched with $500 \mu \mathrm{g} / \mathrm{mL}$ trypan blue, and the GFP fluorescence was quantified using a fluorimeter plate reader (SpectraMaxGemini EM Fluorometer, $485 \mathrm{~nm}$ excitation/535 nm emission, MolecularDevices). Trypan blue served as a blank. A reduction in GFP fluorescence in the killing plate relative to the phagocytosis plate indicated bacterial killing. To examine phagocytosis for E. coli, the Vybrant Phagocytosis Assay Kit (Thermo Fisher Scientific V-6694) was performed following the manufacturer's instructions. Briefly, WT or STAT4-deficient BM neutrophils were incubated with fluorescein-labeled E. coli (K-12 strain) BioParticles (Thermo Fisher Scientific) in complete RPMI media for the indicated time points. Following several wash steps, any membrane-bound, nonphagocytosed bioparticles were quenched with trypan blue. Fluorescein-positive cells were then detected and quantified by FACS.

Neutrophil elastase and Sytox Green assays. Murine neutrophils isolated from BM were stimulated with LPS $(100 \mathrm{ng} / \mathrm{mL})$ or $P$. aeruginosa (10 MOI). Supernatant from each condition was assayed for neutrophil elastase using the Neutrophil Elastase/Ela2 ELISA kit (DY4517-05, R\&D Systems, Bio-Techne). Alternatively, BM neutrophils were isolated from BM and stimulated with LPS (100 ng/mL), MRSA (10:1 MOI), HK-MRSA (10:1 MOI equivalent), or Pam3CSK4 (10 ng/mL) for 60 minutes. Cell-associated neutrophil elastase and 
DNA were determined by staining with specific Ab or Sytox Green (Thermo Fisher Scientific, S7020), respectively, and analyzed by FACS.

Imaging flow cytometry. We applied multispectral imaging flow cytometry to examine characteristics of neutrophils undergoing suicidal and vital NETosis as described (24). Briefly, ex vivo-stimulated BM neutrophils or peritoneum neutrophils from the thioglycolate peritonitis model were tested for changes in cell morphology during NETosis. One million ex vivo neutrophils were stimulated with $1 \mu \mathrm{g} / \mathrm{mL}$ of LPS or $P$. aeruginosa (10 MOI) for at $37^{\circ} \mathrm{C}$ with $5 \% \mathrm{CO}_{2}$. After 1 hour, neutrophils were centrifuged at $500 \mathrm{RCF}$ for 5 minutes at room temperature and resuspended in $100 \mu \mathrm{L}$ of $2 \%$ paraformaldehyde containing 1:1000 diluted Hoechst prior to the acquisition on ImageStream Multispectral Imaging Flow Cytometer (Luminex). For MPO staining, neutrophils were incubated with $0.2 \%$ donkey serum (MilliporeSigma) in PBS for 30 minutes, then incubated with anti-mouse MPO Abs (clone 2D4, Abcam), diluted in blocking buffer for 1 hour at $37^{\circ} \mathrm{C}$, and washed with PBS. Images were acquired on the ImageStream Multispectral Imaging Flow Cytometer, using the $40 \times$ and $60 \times$ magnification objective, which provides a numerical aperture of 0.9 and a pixel dimension of $0.3 \mathrm{~m} \times 0.3 \mathrm{~m}$. A core diameter of $7 \mu \mathrm{m}$ was used in order to minimize in-focus events. The $405 \mathrm{~nm}$ excitation laser was used at an output power of 1 to $10 \mathrm{~mW}$ depending on the intensity of staining. In order to avoid debris and cell aggregate effects, only objects with a minimum cross-sectional area of $50 \mathrm{~m}^{2}$ and a maximum of $600 \mathrm{~m}^{2}$ were collected. Finally, we set up the number of acquiring images to 50,000 cells. All analysis was based on a subsequent report (24). For cell analysis, cell imagery was analyzed using the requisite Image Data Exploration and Analysis Software, version 6.1 (Amnis Corporation). The best focused cells were selected using BF Gradient RMS, a measurement of image contrast that excludes out-of-focus events (area vs. Gradient RMS). Doublets were excluded using SSC Intensity/Hoechst, and only cells with a spot distance of $0-1$ (cells with either single nuclear signal or an elongated signal) were included in the analysis. A Similarity Score that reflects the degree of nuclear translocation of MPO was calculated as described (24). The selected neutrophils were also analyzed for nuclear circularity (nuclear stain, $y$ axis, labeled as Circularity_Morphology: M07, Ch07) versus cell circularity (BF, $x$ axis, labeled as Circularity_Adaptive Erode: M01, Ch01, 95), and 4 different quadrants are representative of each stage of NETosis as identified by changes in the cellular and nuclear morphologies.

Thioglycolate peritonitis model and Sytox Green flow cytometry. WT, Stat $4^{-1-}$, and Stat $4^{l / f l}$ LysM $^{\text {cre }}$ mice were injected with $3 \%$ of thioglycolate broth (Thermo Fisher Scientific) or PBS as a control i.p. to elicit a robust influx of neutrophils into the PC. After 6-72 hours, the peritoneal lavage was collected to investigate the morphological changes and immune responses of neutrophils. A cell-impermeable, DNA-binding Sytox Green dye (Thermo Fisher Scientific, S7020) was used for measuring the NET release. After filtering out the debris with a mesh, neutrophils were analyzed using Attune flow cytometer (Applied Biosystems, Thermo Fisher Scientific). Because Sytox Green expresses fluorescence only after binding to DNA, the step to remove unbound dye can be omitted. FSC and SSC were used to select mainly neutrophils.

MRSA-induced peritoneal infection. The methicillin-resistant S. aureus USA 300 lac strain stocks (provided by Bethany Moore, University of Michigan) were stored at $-80^{\circ} \mathrm{C}$ and cultivated in tryptic soy broth medi-

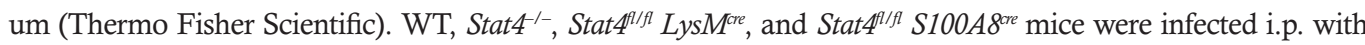
approximately $1 \times 10^{8} \mathrm{CFU}$ of MRSA in $200 \mu \mathrm{L}$ of PBS, and animal survival was monitored until day 10 . For

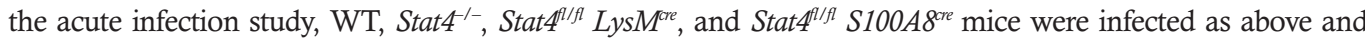
euthanized 16 hours after infection, and peritoneal lavage and the blood were collected to measure bacteria load and analyze peritoneal cell exudate in the recipients using FACS.

Determination of bacterial load. Peritoneal lavage (dilution 1:100/PBS) and blood (no dilution) from WT and Stat $4^{-1-}$, Stat $4^{f l f l}$ LySM $^{\text {cre }}$, and Stat $4^{\text {flfl }}$ S100A $8^{\text {cre }}$ were used to determine bacterial counts. The dilutions were plated on tryptic soy agar medium (Thermo Fisher Scientific), and colonies were incubated at $37^{\circ} \mathrm{C}, 5 \% \mathrm{CO}_{2}$, for 18 hours followed by CFU count. Bacterial burdens were normalized to volume of peritoneal lavage ( $3 \mathrm{~mL} /$ cavity) and calculated by the following equation: (CFU/mL plated) $\times$ (dilution factor) $/$ volume $\mathrm{mL}$. $\mathrm{CFU} / \mathrm{mL}$ represents bacterial burdens in the fluids.

Isolation of BMDMs. BM was harvested from 6- to 10-week-old mice by flushing the femur and tibia bones using cold PBS supplemented with $0.5 \%$ BSA and 2 mM EDTA. Cells were centrifuged at $500 \mathrm{~g}$ for 10 minutes at room temperature. The supernatants were discarded and cell pellets were resuspended in DMEM F12-10 medium, which was supplemented with $10 \mathrm{mM}$ L-glutamine and $100 \mathrm{U} / \mathrm{mL}$ recombinant $\mathrm{M}$-CSF. A total of $2.5 \times 10^{5}$ cells $/ \mathrm{mL}$ were then cultured in $10 \mathrm{~mL}$ sterile plastic Petri dishes and incubated in a $37^{\circ} \mathrm{C}, 5 \% \mathrm{CO}_{2}$, incubator for 3 days and then nourished more with the supplemental DMEM F12-10 until day 7. 
Statistics. For comparisons between 2 groups, unpaired or paired 2-tailed Student's $t$ test was used. Normality was determined using Shapiro-Wilk testing. For comparisons of more than 2 conditions with a defined control group (Transwell experiments), a 1-way ANOVA with post hoc test was used. For all experiments, the means \pm SEM are shown. Significant differences were defined as $P<0.05$.

Study approval. Mice were maintained under specific pathogen-free conditions at Indiana University, Vanderbilt University, and Eastern Virginia Medical School. All experiments were performed with the approval of the IACUCs of Indiana University, Vanderbilt University, and Eastern Virginia Medical School. All aspects of animal research and husbandry were conducted in accordance with the federal Animal Welfare Act and the NIH's Guide for the Care and Use of Laboratory Animals (National Academies Press, 2011). Collection of human peripheral blood was performed with written informed consent and approved by the IRB of Eastern Virginia Medical School.

\section{Author contributions}

EVG, JLN, CHS, and MHK conceived and designed the study. PMB, AKM, WCK, ASN, MH, and MSB conducted experiments. PMB, AKM, PDM, WCK, NSA, ASN, MH, MSB, JLN, CHS, MHK, and EVG contributed to data analysis. EVG and MHK wrote the manuscript, and PMB, AKM, PDM, WCK, NSA, ASN, MH, MSB, JLN, CHS, MHK, and EVG contributed to the manuscript editing.

\section{Acknowledgments}

This work was supported by NIH R01HL142129 (to MHK, JLN, and EVG). This publication was supported by a Project Development Team within the ICTSI NIH/NCRR grant number UL1TR001108 (to MHK) and NIH grants R01HL124159-01, DK122147-01A1, and AI149207A (to CHS). Support provided by the Herman B Wells Center was in part from the Riley Children's Foundation (to MHK). Core facility usage was also supported by IU Simon Cancer Center Support Grant P30 CA082709 and U54 DK106846 (to MHK). ASN was supported by NIH T32 DK007519 and HL007910. AKM was supported by AHA predoctoral fellowship 20PRE35180156. We thank Julia Sharp for providing her expertise for work with human neutrophils.

Address correspondence to: Elena V. Galkina, Department of Microbiology and Molecular Cell Biology, 700 W. Olney Rd., LH3180, Eastern Virginia Medical School, Norfolk, Virginia 23507, USA. Phone: 757.446.5019; Email: galkinev@evms.edu. Or to: Mark H. Kaplan, Department of Microbiology and Immunology, 635 Barnhill Drive, MS 420, Indiana University, Indianapolis, Indiana 46202, USA. Phone: 317.278.3696; Email: mkaplan2@iu.edu. Or to: C. Henrique Serezani, Division of Infectious Diseases, Department of Medicine, Medical Center, 1161 21st Avenue South, Vanderbilt University, Nashville, Tennessee 37232, USA. Phone: 615.322.2035; Email: h.serezan@vanderbilt.edu.

1. Leonard WJ, O'Shea JJ. Jaks and STATs: biological implications. Annu Rev Immunol. 1998;16:293-322.

2. Yamamoto K, et al. Stat4, a novel gamma interferon activation site-binding protein expressed in early myeloid differentiation. Mol Cell Biol. 1994;14(7):4342-4349.

3. Frucht DM, et al. Stat4 is expressed in activated peripheral blood monocytes, dendritic cells, and macrophages at sites of Th1 mediated inflammation. J Immunol. 2000;164(9):4659-4664.

4. Fukao T, et al. Inducible expression of Stat 4 in dendritic cells and macrophages and its critical role in innate and adaptive immune responses. J Immunol. 2001;166(7):4446-4455.

5. Kaplan MH, et al. Impaired IL-12 responses and enhanced development of Th2 cells in Stat4-deficient mice. Nature. 1996;382(6587):174-177.

6. Thierfelder WE, et al. Requirement for Stat4 in interleukin-12-mediated responses of natural killer and T cells. Nature. 1996;382(6587):171-174.

7. Liang Y, et al. Therapeutic potential of STAT4 in autoimmunity. Expert Opin Ther Targets. 2014;18(8):945-960

8. Kaplan MH. STAT4: a critical regulator of inflammation in vivo. Immunol Res. 2005;31(3):231-242.

9. Broxmeyer HE, et al. Th1 cells regulate hematopoietic progenitor cell homeostasis by production of oncostatin M. Immunity. 2002;16(6):815-825

10. Rodriguez-Sosa M, et al. A STAT4-dependent Th1 response is required for resistance to the helminth parasite Taenia crassiceps. Infect Immun. 2004;72(8):4552-4560.

11. Schindler H, et al. The production of IFN-gamma by IL-12/IL-18-activated macrophages requires STAT4 signaling and is inhibited by IL-4. J Immunol. 2001;166(5):3075-3082.

12. Gollob JA, et al. Impairment of STAT activation by IL-12 in a patient with atypical mycobacterial and staphylococcal infections. J Immunol. 2000;165(7):4120-4126.

13. Altare F, et al. Impairment of mycobacterial immunity in human interleukin-12 receptor deficiency. Science. 1998;280(5368):1432-1435. 
14. Godshall CJ, et al. STAT4 is required for antibacterial defense but enhances mortality during polymicrobial sepsis. Clin Diagn Lab Immunol. 2001;8(6):1044-1048.

15. O'Sullivan R, et al. Signal transducer and activator of transcription 4 (STAT4), but not IL-12 contributes to Pseudomonas aeruginosainduced lung inflammation in mice. Immunobiology. 2008;213(6):469-479.

16. Dulson SJ, et al. STAT4 Directs a protective innate lymphoid cell response to gastrointestinal infection. J Immunol. 2019;203(9):2472-2484.

17. Liew PX, Kubes P. The Neutrophil's role during health and disease. Physiol Rev. 2019;99(2):1223-1248.

18. Villarino AV, et al. Mechanisms and consequences of Jak-STAT signaling in the immune system. Nat Immunol. 2017;18(4):374-384.

19. Berdeja J, et al. Phase 2 study of gandotinib (LY2784544) in patients with myeloproliferative neoplasms. Leuk Res. 2018;71:82-88.

20. Hayden MS, Ghosh S. Shared principles in NF-kappaB signaling. Cell. 2008;132(3):344-362.

21. Khajah M, et al. Granulocyte-macrophage colony-stimulating factor (GM-CSF): a chemoattractive agent for murine leukocytes in vivo. J Leukoc Biol. 2011;89(6):945-953.

22. Tecchio C, et al. Neutrophil-derived cytokines: facts beyond expression. Front Immunol. 2014;5:508.

23. Papayannopoulos V. Neutrophil extracellular traps in immunity and disease. Nat Rev Immunol. 2018;18(2):134-147.

24. Zhao W, et al. A novel image-based quantitative method for the characterization of NETosis. J Immunol Methods. 2015;423:104-110.

25. Yang H, et al. New insights into neutrophil extracellular traps: mechanisms of formation and role in inflammation. Front Immunol. 2016;7:302.

26. Lakhundi S, Zhang K. Methicillin-resistant Staphylococcus aureus: molecular characterization, evolution, and epidemiology. Clin Microbiol Rev. 2018;31(4):e00020-18.

27. Deng JC, et al. STAT4 is a critical mediator of early innate immune responses against pulmonary Klebsiella infection. J Immunol. 2004;173(6):4075-4083.

28. Nguyen-Jackson H, et al. STAT3 controls the neutrophil migratory response to CXCR2 ligands by direct activation of G-CSF-induced CXCR2 expression and via modulation of CXCR2 signal transduction. Blood. 2010;115(16):3354-3363.

29. Nutku E, et al. Functional expression of IL-12 receptor by human eosinophils: IL-12 promotes eosinophil apoptosis. $J$ Immunol. 2001;167(2):1039-1046.

30. Collison K, et al. Evidence for IL-12-activated Ca2+ and tyrosine signaling pathways in human neutrophils. J Immunol. 1998;161(7):3737-3745

31. O'Shea JJ, Plenge R. JAK and STAT signaling molecules in immunoregulation and immune-mediated disease. Immunity. 2012;36(4):542-550.

32. Watford WT, et al. Signaling by IL-12 and IL-23 and the immunoregulatory roles of STAT4. Immunol Rev. 2004;202:139-156.

33. Nguyen GT, et al. Neutrophils to the ROScue: mechanisms of NADPH oxidase activation and bacterial resistance. Front Cell Infect Microbiol. 2017;7:373.

34. Ward RA, et al. Priming of the neutrophil respiratory burst involves p38 mitogen-activated protein kinase-dependent exocytosis of flavocytochrome b558-containing granules. J Biol Chem. 2000;275(47):36713-36719.

35. Dang PM, et al. A specific p47phox -serine phosphorylated by convergent MAPKs mediates neutrophil NADPH oxidase priming at inflammatory sites. J Clin Invest. 2006;116(7):2033-2043.

36. Meischl C, Roos D. The molecular basis of chronic granulomatous disease. Springer Semin Immunopathol. 1998;19(4):417-434

37. Zhu Y, et al. NADPH oxidase 2 inhibitor diphenyleneiodonium enhances ROS-independent bacterial phagocytosis in murine macrophages via activation of the calcium-mediated p38 MAPK signaling pathway. Am J Transl Res. 2017;9(7):3422-3432.

38. Romani L, et al. Interleukin-12 in infectious diseases. Clin Microbiol Rev. 1997;10(4):611-636.

39. Cassatella MA, et al. Interleukin-12 production by human polymorphonuclear leukocytes. Eur J Immunol. 1995;25(1):1-5.

40. Goel RR, Kaplan MJ. Deadliest catch: neutrophil extracellular traps in autoimmunity. Curr Opin Rheumatol. 2020;32(1):64-70.

41. Rigby KM, DeLeo FR. Neutrophils in innate host defense against Staphylococcus aureus infections. Semin Immunopathol. 2012;34(2):237-259

42. Butcher MJ, et al. The IL-17A/IL-17RA axis plays a proatherogenic role via the regulation of aortic myeloid cell recruitment. Circ Res. 2012;110(5):675-687.

43. Brandt SL, et al. Macrophage-derived LTB4 promotes abscess formation and clearance of Staphylococcus aureus skin infection in mice. PLoS Pathog. 2018;14(8):e1007244. 